\title{
Ueber die Histogenese der Retina und des Nervus opticus.
}

\author{
Von \\ Dr. Francesco Falchi, \\ Professor an der Universität Cagliari. \\ Mit Tafel I-III.
}

Es ist von grosser Wichtigkeit fur die Biologie, zu wissen, welche zelligen Elemente die erste Anlage eines Organs ausmachen, wie diese Elemente durch ihre Vermehrung das Wachsthum des Organs bewirken und wie sie dann vermittelst der beständig wirkenden Vorgänge der Evolution und Adaptation und durch histochemische Veränderungen die endgültige Gestalt annehmen, welche dem ausgebildeten Organ zukommt. Die vervollkommneten Hilfsmittel, über wèlche die moderne Histologie verfügt, haben unsere Kenntnisse von der Structur der Zelle mächtig gefördert, was von der höehsten Bedentung für die Biologie ist, da ja, wie Virchow richtig bemerkt, ,die Thätigkeit der Organe, welche aus Zellen zusammengesetzt sind, Nichts weiter ist, als die Summe der Thätigkeit aller sie zusammensetzenden Zellen."

Indess haben auch frühere Forscher, mit Mitteln, welche wir jetzt für unztreichend halten, Arbeiten von bedeutender wissenschaftlicher Tragweite zu Tage gefördert. 
So war es bei Untersuchungen über die Histogenese der Retina bereits gelangen, verschiedene Stadien der Entwicklung dieser Membran aufzustellen, welche in Beziehang zu einander gebracht, als Grundlage zu Vorstellungen über die formative Entwicklung der verschiedenen Schichten dieser Membran dienen konnten. Zu den bedeutenderen Arbeiten in der Embryologie der Retina, welche mit derartigen Hilfsmitteln fertig gestellt wurden, gehören die folgenden, deren Ergebnisse ich jetzt kurz auseinandersetzen will.

Babuchin*) zeigte an Embryonen vom Frosch, von Vögeln und Băngetieren, dass die innere Lamelle der secundären Augenblase zusammengesetzt ist aus langen, spindelförmigen Zellen mit Fortsätzen, welche die beiden Oberfächen der Membran erreichen, und aus einer feinen moleculären Intercellularsubstanz. Aus diesen spindelförmigen Zellen sollen sich durch Vermehrung alle anderen Teile der Retina bilden. Die Müller'schen Stützfasern sollen von Zellen abstammen, deren innere Fortsätze dreieckig werden und später die Membrana limitans interna bilden. Kessler*) behauptete dasselbe. Gegen diese Ansicht von Babuchin erklärte sich Arnold." ${ }^{\text {s**; }}$ ) Nach ihm soll die Membrana hyaloidea (welche für die Membrana limitans interna angesehen wurde) von Theilen der Kopfplatten entstehen, welche in Form einer den Glaskörper gegen die Retina abgrenzenden Membran gefaltet würden; darnach soll in einer späteren Zeit eine Vereinigung der Hyaloidea mit der Retina stattfinden.

Nach Babuchin sollen die Ganglienzellen durch Theilung aus denjenigen primitiven Spindelzellen hervorgehen, welche in der Nähe der Fusspunkte der Müller'schen Fasern liegen und die innerste Zellenschicht der Retina bilden. Diese Zellen sollen rund werden, an Volumen zunehmen und mehrere Fortsătze bekommen, von denen der eine sich nach den äusseren Schichten der Retina hin wendet, um dort mit anderen kleinen, wahrscheinlich den zukünftigen Körnerschichten zugehörigen Zellen.

*) Beiträge zur Entwicklungsgeschichte des Auges. Würzburger naturwissensehaftl. Zeitschr., Bd. IV, 1863. - Vergleichende histologische Studien. Ibid., Bd. IV, 1864.

*) Zur Entwicklung des Auges 1877.

****) Beiträge zux Entwicklungsgeschichte des Auges. 1874. 
in Verbindung zu treten. Die Nervenfaserschicht soll zum Theil aus den Fortsätzen der Ganglienzellen, zum Theil aus den Nervenbündeln der Ausbreitung des Sehnerven entstehen. Das Stratum moleculare und das Stratum intergranulare, beide von gleicher Entstehung, sollen eine glänzende, gleichmässige Streifung zeigen; man soll darin spindelförmige Zellen bemerken, welche diese Schichten der Retina abtheilen.

C. Ritter*) fand bei einem 4 Wochen alten mensehlichen Embryo 4 Formen von Zellen: 1. Pigmentzellen der äusseren Lamelle der Augenblase; 2. lange Zellen mit grossen Kernen und centralen Fortsätzen; 3. kleine rundkernige Zellen mit bipolaren Fortsätzen, an der Stelle der inneren Körnerschicht; die äusseren Körner treten später auf; 4. Zellen mit vielen Fortsätzen oder Ganglienzellen. - Bei einem anderen menschlichen Embryo von 10 Wochen fand Ritter die Körnerschichten und die Radialfasern beträchtlich entwickelt und die Nervenzellen relativ klein; mit Ausnahme der M. limitans interna liessen sich sämmtliche Schichten unterscheiden.

$\mathrm{W} u ̈ \mathrm{rzburg} * *)$ fand bei einem $7 \mathrm{~cm}$ langen Kaninchenembryo, dass die Retina das Tapetum, eine Schieht dunkler Elemente, eine Schicht heller Elemente und die Schicht der Nervenfasern zeigte; die letzteren sollen jedoch von Elementen der Retina abstammen und sich in einer bestimmten Ordnung mit den vom Gehirn ausgehenden Fasern des Sehnerven vereinigen. Bei Embryonen höheren Alters sollen die Ganglienzellen durch Umbildung der imeren blassen Zellen entstehen.

Goette ${ }^{* * *}$ ) beobachtete dunkie Kerne in den embryonalen Zellen der Retina des Frosches, welche letzteren sich nicht vollständig in diejenigen Elemente umwandeln, welehe beobachtet werden, wenn diese Membran ibre Entwickelung vollendet hat; diese Zellen sollen in Wirklichkeit ihre Umrisse in der Weise verlieren, dass ihre Zellkörper sich mit einander vereinigen; darauf umgeben die Kerne sich mit einem Theil dieser Masse

*) Zur histologischen Entwicklungsgeschichte des Auges. Archiv f. Ophthalm. Bd. X, Abth. 1, p. 60. - Zweiter Beitrag zur Histogenese des Auges. Ibid., Bd. X, Abth. 2, p. 142. 1864.

*) Beiträge zur Bildungsgeschichte der Iris und der Retina beim Kaninchen. Med. Centralbl. 1875, p. 820. - Zur Entwickelungsgeschichte des Säugethierauges. Arch. f. Augen- und Ohrenheilkunde Bd. V, Abth. 2, p. 251.

***) Die Entwickingsgeschichte der Unke. 1875. 
und bilden auf diese Weise die neuen Zellen, während aus dem Rest derselben die Intercellularsubstanz entstehen soll.

Löwe*) fand bei Kaninchenembryonen die embryonalen Zellen der Retina gross, länglich, rund, mit hervortretendem Kern und spärlichem Protoplasma; er stellte zwei Formen von Zellen fest; er bemerkte, dass einige embryonale Zellen nicht. in der ganzen Dicke der Retina zu finden sind und beschrieb 6 Lagen von Zellen in der Papille.

Nach $0 \mathrm{gneff}$ ) besteht die Retina eines $4-5 \mathrm{~mm}$ langen Kaninchenembyro aus spindelförmigen Zellen mit feinen Fortsätzen, welche die ganze Retina durchsetzen. Später werden die innern Zellen grösser, rund und teilen sich; die grösste Zahl der Fortsätze verläuft nach innen, biegt um, läuft parallel mit der Retina und bildet so die Schicht der Nervenfasern. Die mehr nach aussen gelegenen Zellen schicken nur Fortsätze nach dem Innern und bilden die kleinen Elemente, welche die Anlagen der Müller'schen Radialfasern sind. In der Folge erseheint als schmaler Streifen die Anlage der Molecularschicht, welche aus Nervenzellenausläufern, Radialfasern und dazwischen gestrenten feinen Körnchen besteht. Später sollten darin freie Kerne und runde Zellen zu finden sein. Die Differenzirung der beiden Körnersehichten soll durch eine Theilung in der äusseren Zellenschicht stattfinden.

Kölliker ***) bemerkte bei $8-8,5 \mathrm{~mm}$ langen menschlichen Embryonen, dass das distale Blatt der secundären Angenblase in seiner ganzen Länge aus länglichen, in 4-6 Lagen angeordneten Zellen besteht. Bei einem menschlichen Embryo von $15 \mathrm{~mm}$ Länge zeigt die distale Membran in ihrem inneren Theil eine dünne Schicht von Zellen mit runden Kernen und in ihrem äusseren Theil eine dicke Schicht von Zellen mit länglichen Kernen; zwischen diesen beiden findet sich eine helle zellenarme Schicht; ausserdem findet sich in der distalen Lamelle ein feiner Streifen von Opticusfasern ohne alle Zellen. Bei einem menschlichen Embryo von $21 \mathrm{~mm}$ Länge endlich fand Kölliker, dass die distale Lamelle aus zwei Zonen bestand, einer hinteren mit sehr deutlichen Fasern des Nervus opticus und mit der

*) Ueber die Histogenese der Retina. Arch. f. mikrosk. Anatomie X V, 1878.

* Centralblatt f. d. med. Wissenschaften. No. 35, 1881.

***; Zur Entwicklung des Auges und Geruchsorganes menschlicher Embryonen. 1883. 
Differenzirung in verschiedene Schichten, die Külliker schon vorher beschrieben hatte (eigentliche Retina), und einer vorderen von ganz gleichmässigem Bau (Pars ciliaris). ${ }^{*}$ )

Was die weitere Entwicklung der Retina betriff, so wiesen Babuchin und Ogneff die Molecularsehicht in Mitten der Schicht der Spindelzellen nach, welche die Schicht der Ganglienzellen abtrennen sollte. Während seiner Bildung soll das Stratum intergranulare aus Spindelzellen bestehen, welche in der inneren und äusseren Körnerschicht reichlich vorhanden sind, und es sollte sich in derselben Weise, wie das Stratum moleculare internum entwickeln; bei den Kaulquappen sollen diese beiden Schichten zu gleicher Zeit auftreten, während beim Hühnchen die innere Molecularschicht früher entstehen soll. Aus den übrigen Primitivzellen sollen sich die anderen Schichten der Retina bilden, welche, ron Aussen nach Innen gezählt, folgende sind: Die Schicht der Innenglieder der Stäbchen und Zapfen, die Membrana limitans externa, die innere Körnerschicht; doch wird diese bei Kaninchen erst nach der Geburt fertig. Sechs Wochen später haben die Netzhautschichten die histologische Structur, welche man bei diesen Thieren in ausgewachsenem Zustand antrifft.

Nach Ritter **) liessen sich in der Retina eines 10 wöchentlichen menschlichen Embryo das Innenglied und das Aussenglied der Stăbchen und Zapfen unterscheiden. Sie zeigten einen centralen fadenförmigen Fortsatz, welcher bis in das Innere des Stäbchens hinein zu verfolgen war.

Goette***) beobachtete, dass die Stäbchen und Zapfen beim Frosch nicht als Fortsätze runder Zellen auftreten (Babuchin), sondern vielmehr als bläschenförmige Enden länglicher Zellen, welche gegen die Pigmentsehicht vordringen und von dieser ihre Decke erhalten. Nach Loewe †) soll die Entwicklung der Innenund Aussenglieder der Stäbchen und Zapfen unabhängig ron einander erfolgen. Sie beginnt mit dem Hervortreten des Aussengliedes und endigt mit der Entwicklung des Innengliedes. Nach Kupfert门) würde die Entwicklung des Anssen- und Innengliedes

*) Entwicklungsgeschichte des Mensehen und der höheren Thiere. 2. Aufl., p. 693 .

***) l. c.

***) l. c.

†) l. c.

f†) Die Entwicklung der Retina des Fischauges. Centralbl. f. d. med. Wissensch. No. 41, 1868. 
Fr. Falchi.

der Stäbchen und Zapfen in der ersten Periode getrennt vor sich gehen. Kölliker*) zeigte beim Bombinator, dass die Stäbchen und Zapfen aus einer Umbildung der Zellon der äusseren Körnerschicht hervorgehen. Dasselbe wurde von Babuchin bei Kaulquappen und von Waldeyer bestätigt, später anch von IN. Schultze, W. Krause, Schenk, Förster und Balfour, N. Lieberkühn and Ogneff anerkannt.

W. Maller**) hält, wie zuerst auch M. Schultze und W. Krause die Stäbchen und Zapfen für cuticuläre Gebilde. Nach M. Schultze ist beim Hühnehen, beim Menschen und bei den Wiederkäuern die Stäbchen- und Zapfenschicht bereits vor beendetem Fötalleben entwickelt; die Neugeborenen, welche blind (d. h. mit geschlossenen Lidern) zur Welt kommen, sollen noch keine Spur von Stäbchen zeigen; das gelte sowohl für das Kaninchen als für die Katze. Hiermit stimmt Krause in Bezug auf die Katze nicht überein.

Bezüglich der Histogenese des Tapetum nigrum glaubt die grösste Zahl der Autoren, dass es von dom äusseren Blatt der secundären Augenblase abstamme, während aus dem inneren Blatte sich die übrigen Schichten der Retina entwickelten. Diese Ansicht wurde zuerst von E. Huschke ****) ausgesprochen und dann von Schöler $\dagger$ ), von Remak + ), A. Müller $\left.+t_{\dagger}\right)$, Kölliker*t), Babuchin**t), Max Schultze**t), Schenkt*), Kupfer $t^{*}$ ) angenommen.

*) Mikr. Anatomie. II. p. 729, Fig. 424f.

* Ueber die Stammesentwicklung des Sehorgans der Wirbelthiere. Festschrift an C. Ludwig, 1875.

*\%) S. Th. v. Soemmering's Lehre von den Eingeweiden. Leipzig 1844. p. 714.

†) De oculi evolutione in embryonibus gallinaceis. Dissert. inaug. Dorpati Liv., 1848, 4.

f) Untersuchnngen über die Entwickelung der Wirbelthiere. Berlin 1855.

†t) Die Entstehung der Retina. Allgem. med. Centralzeitung. Berlin 1858. p. 361.

f) Entwickelungsgeschichte des Menschen und der höheren Thiere. Leipzig 1861. 8.

$*$ t) 1. c., p. 71 .

****) Zur Anatomie und Physiologie der Retina. Archiv für mikrosk. Anatomie Bd. II, p. 236.

†) $1 . \mathrm{c}$.

t+ $1 . \mathrm{c}$. 
Hensen*) ist der entgegengesetzten Meinung wie Huschke. Schöler betrachtet die Aussenglieder der Stäbchen und Zapfen und besonders das Tapetum nigrum als Abkömmlinge der äusseren Wand der secundăren Augenblase. Max Schultze ${ }^{* *}$ ) stimmt nicht mit Hensen überein und behauptet bei einem Hühnchenembryo von 80 Stunden beobachtet zu haben, dass sich sehwarzes Pigment in der ausseren Schicht des äusseren Blattes ablagerte, welche in dieser Epoche (6ter Tag) aus langen prismatischen Zellen besteht, welche später kürzer werden. Babuchin***) und Schenk $\dagger$ ) fanden, dass bei niederen Wirbelthieren die äussere Lamelle der secundären Augenblase ans einer einzigen Lage von Zellen besteht, während Kölliker $t_{\dagger}$ ) geneigt ist, beim menschlichen Embryo zwei Zellenschichten zu zeichnen. Doch fand Köllik er $+\dagger$ ) selbst vor einigen Jahren, dass bei 8-21 $\mathrm{mm}$ langen menschlichen Embryonen das Tapetum aus 2 und an einigen Stellen aus 4-5 Lagen cubischer polygonaler Zellen gebildet wird. Bei den Săugethieren beginnt die Pigmentbildung zum Theil gleichzeitig mit der Abschnürung der Linse, zum Theil später. Die Pigmentkörner treten bei diesen Thieren wie beim Menschen zuerst in den tieferen Parthien der Zellen, d. h. nach der Höhlung der primitiven Augenblase zu, auf und die Pigmentirung geschieht in den hinteren Partien der Retina früher als in den vorderen. Von dort sebreitet die Pigmentirung nach W ürzburg*io ziemlich schnell mehr oder weniger weit auf das distale Blatt fort, bevor noch die Iris sich entwickelt.

Nach der Faltung der primitiven Augenblase ist der Stiel aus mehr Zellenlagen zusammengesetzt, als die fötale Retina (Hensen); Husehke, Schöler und A. Müller lassen den

*) Zur Entwickelung des Nervensystems. Virchow's Arch. f. path. Anatomie Bd. XXX, p. 76, 1864. - Ueber den Bau des Schneckenauges und über die Entwickelung der Augentheile in der Thierreihe. Areh. f. mikroskop. Anatomie Bd. II, p. 399, 1866.

**) l. c.

**; $1 . \mathrm{c}$.

†) $1 . \mathrm{c.}$

ti) l. o.

HH Zur Entwickelung des Auges und des Geruchsorganes menschlicher Embryonen. 1883.

*) $1 . \mathrm{e}$. 
Fr. Falchi.

Sehnerv aus der inneren Oberfläche des Stiels und dem mit der A. centralis retinae eingedrungenen Bindegewebe entstehen.

His*) and Köllilker**) sind der Ansicht, dass der Norvus opticus der Wirbelthiere als ein Theil des Gehirns angesehen werden müsse und der Stiel der primitiven Augenblase sich in indifferentes Stutzgewebe umwandele, welches den Nervenbündeln, die sich vom Gehirn in die Retina hineinbegeben, zur Führung diene. His ist der Ansicht, dass die Nervenfasem durch ein Längenwachsthum der Ganglienzellen entstehen; da nun der* Stiel der Augenblase keine Ganglienzellen enthält and die Ganglienzellen des Gehirns hier die Nervenfasern bilden, so müssten die Nervenfasern des Optictis rom Gehirn ausgehen und auf dem Wege des Augenblasenstiels die Retina erreichen.

Die mittelst des Stieles im Anfang hergestellte Zellenverbindung zwischen Augenblase und Gehirn wird nach His später wieder dadurch unterbrochen, dass die Zellen des Stieles wieder in das Gehirn zurücktreten.

Gegenüber der His'schen Theorie leitet W. Müller die Fasern des Nervus opticus von den Ganglienzellen der Retina $a b$, von der aus die Fasern eine centripetale Richtung nehmen sollen, um in das Gehirn einzudringen.

Lieberkühn***) behauptet His gegenüber auf das bestimmteste, dass der Sehnerv aus dem primordialen Stiel der Augenblase entstehe und der primordiale Sehnerv nur von seinen eigenen Zellen her seino Nervenbündel und sein Stützgewebe besitze. Das Gewebe des N. opticus bestehe im ersten Stadium aus denselben mit Kernen versehenen, radiär angeordneten Spindelzellen, wie das Gehirn selbst. Später soll sich erst eine feine Längsstreifung unter gleichzeitiger Verminderung der Radialstreifung entwickeln, ausser den feinen Fasern sollen sich zahlreiche mit wenig Protoplasma umgebene Kerne finden, welche isolirt als spindelförmige Körper erschienen. Ein Theil der Zellen soll die Nervenfasern bilden, welche in Zusammenhang

*) Untersuchungen über die erste Anlage des Wirbelthierleibes. Die erste Entwicklung des Hühnchens im Ei. Leipzig 1868. 4. 12 Tafeln.

**) Entwickelungsgeschichte etc.

***) Ueber das Auge des Wirbelthierembryo. Schriften der Marburgex Gesellsch. zur Beförderung der Naturwissenschaften Bd. X, p. $299-381,1872$. 
mit dem Gehirn ständen, welches an der Peripherie eine faserige Structur besitze. Manz*) theilt diese Ansicht.

C. K. Hofmann***) hat beobachtet, dass der Nervus opticus der Knochenfische durch eine Umbildung der Zellen des Stiels gebildet wird. Doch würde für die Klasse der Wirbelthiere die Anschauung von His die Regel bilden.

Nach Mihalcowics***) soll die Entwicklung der Nervenfasern des N. opticus wahrscheinlich in centrifugaler Richtung stattfinden. Die bindegewebigen Elemente des $\mathbf{N}$. opticus sollen vom mittleren Keimblatt abstammen und nicht, wie W. Müller will, von den Bildungszellen des Sehnerven.

Bergmeister $\dagger$ ) bekennt sich im Allgemeinen zu der Ansicht von His bezüglich der Fntwicklung des Sehnerven und bezeichnet den Stiel der Augenblase als Gubernaculum für die aus dem Gehirn kommenden Nervenbündel. Er wollte an einem Horizontalschnitt des Angenblasenstiels das Auftreten eines zapfenförmigen Gebildes constatirt haben, welches mit dem inneren Blatt der Augenblase in Verbindung stände. Der Zapfen soll durch Hineinwachsen des Mesoderms erzengt sein, welehes den Hohlraum oder Canal ausfüllt, der in Folge der Einstülpung des vorderen, mit der Retina in Verbindung stehenden Theils des Opticusstiels entstanden ist.

Nach Kolliker $\dagger$ ) ist der Nerrus opticus bei menschlichen Embryonen von 8-15 mm Lange hohl, zeigt eine deutliche Einstülpung und seine Wände haben die gleiche Structur wie das Vorderhirn.

Bei einem menschlichen Embryo von $20 \mathrm{~mm}$ bestand der N. opticus aus einem Stroma sternförmiger Zellen mit feinen Optieusfasern.

Hiltner $\uparrow^{\dagger} \dagger$ ), welcher seine Studien an der Haus- und Feld-

*) Entwicklangsgeschichte des Anges, Graefe und Saemisch, Handbuch der gesammten Augenheilkunde Bd. II, Cap. 5.

**) Zur Ontogenie der Knochenfische. Arch. f. mikrosk. Anatomie Bd. XXIII, Heft 1.

***) Entwicklungsgeschichte des Gehirns. p. 195.

†) Zur Entwicklungsgeschichte des Sängethierauges. Mittheilungen des embryolog. Instituts in Wien Heft 1, p. 63.

it) Zur Entwickelung des Auges und Geruchsorganes menschlicher Embryonen. Würzburg 1883.

†t十) Ueber die Entwicklung des Nervus opticus der Sängew thiere. Biolog. Centralbl. Bd. V, p. 186. 
maus und am Meerschweinchen machte, erklärt sich gegen die Theorie von His und von Kölliker: nach ihm dringt nach vollendeter Invagination des inneren Theils der Augenblase und der Linse zwischen diesen beiden eine feine Mesodermaschicht ein. Im Stiel finde eine Einstülpung nicht statt, sondern vielmehr eine Vortreibung eines Theils der Retina, welcher wie ein länglicher Zapfen in den Opticusstiel hineinwachse.

Die Umbildung der Zellen in Nervenfasern beginne immer in der inneren Schicht der Retina und ihres Zapfens gleichzeitig mit der Entwickllung des Chiasma. Darnach würden die Bündel des Nerrus optiens nicht vermittelst einer Proliferation des peripheren Gehirntheiles gebildet, sondern ein autochthones Gebilde darstellen.

Nach Kuhnt*) soll das Dickenwachsthum des Nervus opticus nicht in einer Vermehrung, sondern in einem Breiterwerden der Nervenfasern bestehen.

Was die Scheide des Opticus angeht, so soll sich dieselbe nach Kölliker***) wie die Sclerotica aus dem Mesoderm bilden und bei 2 monatlichen menschlichen Embryonen sichtbar werden.

Nach der geschilderten Periode der histologischen Untersuchungen der Retina begann man vor einigen Jahren mit vollkommneren Mitteln der mikroskopischen Technik die Histogenese der Retina zu untersuchen and die durch Karyokinese erfolgende Theilung ihrer Elemente zu erforschen.

Kog an $\theta \mathrm{i}^{*}$ **) unterscheidet bei Hühnerembryonen in der Wand der primären Augenblase zwei Schichten, eine änssere, aus Spindelzellen zusammengesetzte und eine innere blassere, deren Zellen karyokinetische Figuren zeigen, weshalb der Autor sie proliferirende Zellen nennt. Dieselben findet man, wenn später die secundäre Augenblase gebildet ist, in der äusseren Oberfläebensehicht der distalen Lamelle der Retina. Es ist dies die Zeit, in welcher die distale Lamelle in die Dicke zu wachsen,

*) Zur Kenntniss des Sehnerven and der Netzhaut. Aroh. f. Ophthalm. Bd. XXV, Abth. 3, p. 179.

*) Entwickelungsgeschichte etc.

***) Untersuchuagen über die Histogenese der Retina. Arehiv f. mikrosk, Anat. Bd. XXII, Heft 3, p. 342. 
die proximale dünner zu werden beginnt. In diese äussere Schicht der distalen Lamelle mit in Karyokinese begriffenen Zellen verlegt Koganei den ganzen Entwicklungsvorgang der Retina, weil er hier allein eine Karyokinese der Elemente antraf. Diese proliferirenden Zellen sollen verschwinden, sobald die Stäbchen zu erscheinen anfangen. Die Karyokinese soll mit radialer oder tangentialer Theilungsebene auftreten. Er nimmt die Zusammensetzung aus Spindelzellen nicht für die ganze Dicke der distalen Lamelle an, wie Babnchin und $0 \mathrm{gneff}$, sondern beschreibt an der inneren Oberläche der distalen Lamelle ein feines Netzwerk mit grossen runden Zellen mit Kernen und Kernkörperehen und wenig Protoplasma, welche später zu Ganglienzellen werden sollen. Die proximale Lamelle besteht aus einer Reihe von Zellen, die in ihrem äusseren Theil Pigment besitzen.

Bei 5 Tage alten Hühnerembryonen zeigt nach ihm die Retina 5 Sehichten: 1. Membrana limitans interna; 2. die Schicht der Opticusfasern; 3. die Ganglienzellenschicht; 4. die Primitivzellenschicht; 5. das Stratum proliferans. Am 7 . Tage erscheint das Stratum moleculare und am 11. die erste Spur der M. limitans externa. Aus den Zellen der äusseren Körner entwickeln sich die Zapfen und Stäbchen. Am 17. Tage soll die Histogenese der Retina des Hühnchens vollendet sein.

Bei Säugethieren (4 $\mathrm{mm}$ langen Kaninchenembryonen) hat die primäre Augenblase zwei Schichten von Zellen: die primitiven und die proliferirenden Zellen.

Bei Embryonen von $5 \mathrm{~mm}$ Länge stülpt sich die Augenblase ein; bald zeigt das äussere Blatt eine Reihe cubiseher Zellen, welche Pigmentkörner enthalten; die distal $\theta$ Lamelle theilt sich in zwei Schichten, eine mit Zellen und eine andere aussere ohne Zellen. Bei Embryonen von $12 \mathrm{~mm}$ erscheinen die Nerrenbündel in der Nähe des inneren Pols; sie stehen in Verbindung mit den Ganglienzellen. Später tritt das Stratum reticulare mit seiner netzförmigen Structur hervor.

Die Theilung der beiden Körnerschichten durch das Auftreten der Zwischenkörnerschicht findet in den letzten Tagen vor der Geburt statt. In den beiden ersten Wochen nach der Geburt ist die Struetur der Retina vollendet. Bei andern Säugethieren (Schwein, Schaf und Katze) beobachtete Koganei ähnliche Verhältnisse. Doch sollen beim neugebornen Heerschweinchen die Stäbchen und Zapfen bereits entwickelt sein. 
Ich fand $*$ ) gleichzeitig bei Untersuchungen über die Histogenese der Retina und des $\mathbf{N}$. opticus an Säugethierembryonen (rom Rind, Hund, Meerschweinchen) in Karyokinese begriffene Zellen nicht nur in der äusseren Oberflächenschicht der distalen Lamelle der secundären Augenblase von $4,7 \mathrm{~cm}$ langen Rindsembryonen, wie auch Koganei in dieser Schicht bemerkt hatte, sondern auch in den übrigen Elementen der anderen Schichten dieser distalen Lamelle. Doch waren die Zellen mit Mitosen in der äusseren Oberflächenschicht derselben zahlreicher als in ihren anderen Schichten. Bei 7,5 cm langen Rindsembryonen habe.ich die Mitose an den Zellen, welche zwischen den Nervenfasern der Ausbreitung des $\mathrm{N}$. opticus gelagert sind, beobachtet, wenn auch der karyokinetische Process viel häufiger an den im Stamm des Nerven selbst, zwischen den Nervenfasern liegenden Zellen zu finden ist. - Abgesehen von der Retina und dem $N$. opticus trifft man die Karyokinese auch in den Zellen des Mesodermas, welches die Anlage der Sclerotica bildet, bei Rindsembryonen von $7,5 \mathrm{~cm}$, Hundeembryonen von $15 \mathrm{~cm}$ und Meerschweinchenembryonen von $8 \mathrm{~cm}$.

Merk**) sah bei Embryonen von Trepidonotus natrix, dass die den Ventrikeln zugekehrte Oberfläche des Räckenmarkscanals Mitosen zeigt; in gleicher Weise fand er die Karyokinese in den Zellen der äusseren Schicht des distalen Blattes. Daraus folgert Merk, dass das Wachsthum der Retina an der äusseren Oberfläche ihres distalen Blattes vermittelst der indirekten Theilung beginnt. In der Folge sollen sich zuerst die Ganglienzellen bilden und die Fasern des N, opticus, das Stratum moleculare externum und die innere Körnerschicht differenziren; die anderen Schichten sollen entstehen, wenn die Zapfen sich bildon und hierauf soll die Karyokinese aufhören. Die Mitosen waren in radialer Ebene angeordnet. Desgleichen beobachtete Merk die Mitose bei einem 2,5 cm langen Kaninchenembryo an demselben Orte, wo er sie bei Trepidonotus natrix gefunden hatte.

*) Gazzeta delle Oliniche, anno 1886, p. 342. - Annali d'Ottalmologia Anno XV, Fasc. 1, p. 100.

*) Ueber die Anordnung der Kerntheilungsfiguren im Centralnervensystem and der Retina bei Natternembryonen. Sitzungsberichte der k. k. Akademie der Wissenschaften in Wien Bd. XCIT, Abth. Oct.-Heft, Jahrg. 1885. 
Rauber*) erklärt bei Froschembryonen die äussere Zellenschicht der distalen Retinallamelle als Praedilectionsschicht für die Mitose; ausserdem fand er aber auch Mitosen an den Zellen der zweiten Schicht (von aussen gerechnet) und in denen der mittleren Schicht der distalen Retinalamelle. Die Theilungsebenen bei den ausseren Mitosen sind zum Theil tangential, zum Theil radial, doch sind diagonale Theilungsebenen auch nicht selten.

Bei der Fortsetzung **) meiner Untersuchungen über die Histogenese der Retina bei Säugethierembryonen (Rind, Kaninchen, Meerschweinchen, Hund) erhielt ich ausser der Bestätigung von dem, was ich in meiner ersten Mittheilung auseinandergesetzt habe, folgende neue Ergebnisse:

1. In der distalen Lamelle der Retina findet man die Karyokinese in allen ihren Regionen, d. h. sowohl am hinteren Pol, als am Aequator, als in der Ciliarportion und in Zellen der verschiedenen Schichten dieser Lamelle;

2. In der hellen Innenzone der distalen Retinallamelle beobachtet man bei $7,5 \mathrm{~cm}$ langen Rindsembryonen und bei $4,3 \mathrm{~cm}$ langen Kaninchenembryonen Zellen mit grossen, schwach gefärbten Kernen, dagegen bekommen diese Zellen ausgeprägtere Charaktereigenschaften bei Rindsembryonen von $8 \mathrm{~cm}$ Länge.

3. Das Tapetum nigrum wird bei Rindsembryonen von 1,8 bis $2,4 \mathrm{~cm}$ Länge aus (in der Seitenansicht) cubischen Zellen gebildet und zwar sowohl am hinteren Pol als am Aequator; dieselben zeigen eine Theilung durch Karyokinese; in dem vorderen Theil haben die Zellen des Tapetum dieser Embryonen eine ausgeprägt cylindrische Gestalt, zeigen aber auch die Karyokinese. Doch haben Zellen dieser proximalen Lamelle (in der Ansicht von vorn) bei Rindsembryonen von $6,2 \mathrm{~cm}$ und Kaninchenembryonen von $4,3 \mathrm{~cm}$ Länge eine beinahe polygonale Form und zwar sowohl am hinteren Pol, als am Aequator.

4. Das erste Zeichen des Beginns der Entwicklung des Uvealtractus bei Sängethierembryonen, welche von mir untersucht wurden, ist eine reiche Mitose in den Zellenelementen

*) Die Kerntheilungsfiguren im Medullarrohre der Wirbelthiere. Arch. f. mikrosk. Anatomie Bd. XXVT, 4. Heft, p. 623.

**) Annali d'Ottalmologia, Anno XV, Hasc. 5 u. 6, Rendiconto del Congresso in Genova della Società Ottalmologica Italiana nell' octobre 1886, p. 93. 
seiner mesodermalen Anlage, besonders in den den Zellen des Tapetum nigrum zunächst gelegenen Elementen.

Meine Untersuchungen uber die Histogenese der Retina und des Nervus opticus wurden an Sängethierembryonen (Kaninchen, Rind, Hund, Meersehweinchen, Mensch) angestellt. Die Augen der Embryonen wurden in Alkohol gehärtet. Die Färbung der Schnitte geschah mittelst der Methode von Bizzozero*), mit Grenacher's Alauncarmin und mit Hämatoxylin.

Primäre und secundäre Augenblase.

Die primäre Augenblase ist, wie schon E. v. Baer**) zum ersten Male gezeigt hat, ein Theil der vorderen Hirnblase. Die primäre Augenblase erfährt später eine Einstülpung, indem sich ihre vordere Wand nach innen einbiegt, wodurch die secundäre Angenblase entsteht, welche daher aus einem äusseren Blatte, der sogenannten proximalen Lamelle, die zum Tapetum wird, und einem inneren Blatte, der distalen Lamelle, aus der die eigentliche Retina entsteht, zusammengesetzt ist. Es steht daher in dieser Entwicklungsperiode das äussere Blatt der securiären Augenblase durch den Augenblasenstiel, welcher die Anlage des Nervus opticus darstellt, mit der vordern Hirnblase in Verbindung.

Bei Kaninchenembryonen von 10 Tagen hat sich die secundäre Augenblase schon gebildet und ihr Stiel, die Anlage des Nervus opticus, besteht ans 5 Lagen radial angeordneter Zellen mit runden oder ovalen Kernen. Die runden oder wenig ovalen Kerne zeigen ein gefürbtes Netzwerk und einen farblosen, das Licht reflectirenden Kernsaft; dagegen sind die anderen Kerne deutlicher oval, ihr Netz-

*) Neue Methode, die in Kerntheilung begriffenen Zelien in den Geweben nachzuweisen. Zeitschr. f. wissenschaftl. Mikroskopie Ba. III, Heft 1, p. 24.

**) Ueber Entwickungsgeschichte der Thiere etc. I. Theil 1828; II. Theil 1837. 
werk ist intensiv gefärbt und auch ihr Kernsaft ist es ein wenig, wie dies meistens bei Zellen des Mesoderma der Fall ist. Die Zellen mit mehr ovalem Kern sind hier weniger zahlreich als in dem Mesoderma, während die mit runden oder wenig ovalen Kernen zahlreicher sind. Die Zellen der Innenfläche des Stiels zeigen viele Kerntheilungsfiguren (Fig. 1 a).

Diese selben Zellen, wie sie eben im Stiel beschrieben wurden, bemerkt man auch in der proximalen Lamelle der secundären Augenblase und zwar am hinteren Pole und am Aequator in 4-6 Lagen angeordnet, während sie in ibrem vorderen Theil deren $4-5$ zeigt. Auch hier bemerkt man in der proximalen Lamelle zahlreiche Mitosen in den Zellen ihrer Innenfläche (Fig. 1b), welche das Lumen der primären Augenblase nach Aussen hin begrenzt.

Die distale Lamelle hat an ihrem hinteren Pole die Gestalt einer etwas unregelmässigen Kegelspitze, welche sich in der innern Oeffnung des Canals oder dem innern Hohlraum zwischen den Wänden des Stiels oder des Nervus opticus präsentirt; hier besteht die distale Lamelle aus 6-7 Lagen von Zellen, welche denen der proximalen Lamelle des Stieles und der vorderen Hirnwand ähnlich sind: Am Aequator zeigt sie 4-5 Zellenlagen; an ihrem vorderen Rande, wo sie in die proximale Lamelle übergeht, zählt man deren 4. In den Zellen der distalen Lamelle beobachtet man zahlreiche Karyokinesen in den Zellen der äusseren Oberflächenlage (Fig. $1 \mathrm{c}$ ), welche der proximalen gegenübersteht und so nach Innen zu den Raum der primären Augenblase begrenzt.

Im Allgemeinen sind in der proximalen und distalen Lamelle und in den Wandungen des Sehnervenstiels die Zellen mit runden oder etwas ovalen Kernen zahlreicher, während die anderen Zellen mit stärker ovalen Kernen hier in geringerer Anzahl vorhanden sind.

v. Graefe's Archiv für Ophthalmologie, XXXIV. 2. 
Aus dieser Beschreibung ergiebt sich, dass in dieser Entwickelungsperiode sowohl die Wandungen des Sehnervenstiels, als die proximale und die distale Lamelle eine unter sich und mit der Wand der vorderen Hirnblase übereinstimmende Structur darbieten.

In dem Mesoderma, welches die secundäre Augenblase umgiebt, zeigt die Mẹhrzahl der Zellen ovale Kerne, deren Netzwerk intensiv gefärbt und deren Kernsaft auch etwas gefärbt ist; doch finden sich auch hier Zellen mit runden oder leicht ovalen Kernen mit wenig gefärbtem Netzwerk und blassem Kernsaft.

In allen diesen Zellen des Mesoderma finden sich zahlreiche Mitosen (Fig. $1 \mathrm{~d}$ ).

\section{Histogenese der Retina.}

1. Histogenese der eigentlichen Retina.

Bei der weiteren Entwickelung der secundären Augenblase beginnt deren proximale Lamelle dünner zu werden, während die distale anfängt, in die Dicke zu waehsen.

Hiernach besteht die dicker gewordene distale Lamelle (bei $18 \mathrm{~mm}$ langen Rindsembryonen) an ihrem hinteren Pole aus Zellen mit meistens ovalen (Fig. 2, A a) manchmal auch runden Kernen, mit gefärbtem Reticulum und Kerninhalt, doch ist der Nucleolus immer intensiver gefärbt; diese Zellen sind radienformig in 8-10 Lagen angeordnet. Wahrend sich die Zellen mit ovalem Kerne in der ganzen Dicke der distalen Lamelle finden, bemerkt man die Zellen mit rundem Kern in der äusseren Oberflächenschicht und am inneren Rande der Lamelle.

In den Zellen der äusseren Oberflächenschicht giebt es viele Karyokinesen mit tangentialer Theilungsebene, doch macht sich die Mitosis auch noch in den Zellen bemerkbar, welche in der dritten Zone, von aussen gerechnet, gelegen sind (Fig. 2, A b). Die Zellen der distalen Lamelle 
liegen meistens dicht aneinander, nur gegen den inneren Rand der Lamelle hin erscheinen sie weiter auseinander gerückt. Diese Zellen schicken blasse Fortsätze nach innen und nach aussen, welche zwei weissliche Streifen bilden, und zwar ist der innere derselben breiter als der äussere, welcher die distale Lamelle begrenzt. 'Am Aequator besteht diese Lamelle aus $7-8$ Lagen der beschriebenen Zellen in gleicher Anordnung. Sowohl in dem äquatorialen als in dem vorderen Theile der distalen Lamelle bemerkt man ebenfalls in Karyokinese begriffene Zellen mit radialer Theilungsebene in der 2., 3. und 4. Lage, vom äusseren Rande aus gerechnet. In ihrem vorderen Theile zeigt die distale Lamelle eine von hinten nach vorn fortschreitende Verminderung ihrer Zellenlagen von 6 bis 2 . In dem Ciliartheil vermindern sich dieselben von 3 auf 2 Lagen.

Aber sehr bald bekommt die distale Lamelle bei fortschreitender Entwicklung eine grössere Dicke; an ihrem binteren Pole besteht sie aus 9-12 Lagen von Zellen mit länglich ovalem Kerne, mit intensiv gefärbtem Reticulum, während der Kerninhalt nur leicht gefärbt ist (bei $1,8 \mathrm{~cm}$ langen Kaninchenembryonen), aus 10-14 Lagen bei 2 bis $5,2 \mathrm{~cm}$ langen Rindsembryonen. In der Nähe der inneren und äusseren Oberfläche der Lamelle zeigen die Zellen auch runde und leicht ovale Kerne mit gefärbtem Netzwerk und 1) assem Kernsaft. In allen an der äusseren Oberfläche gelegenen Zellen bemerkt man zahlreiche Mitosen (Fig. 4a), meistens mit tangentialer, manchmal aber auch mit radialer Theilungsebene; doch beobachtet man die Mitosen mit radialer oder tangentialer Richtung in gleicher Weise auch in den medianen Schichten (Fig. $4 \mathrm{~b}$ ) wie in den anderen mehr inneren Schichten in der Nähe der Nervenfibrillenbündel. Ein heller, blasser, aus den nach aussen gerichteten Fortsätzen der Zellen der distalen Lamelle gebildeter Saum begrenzt diese Membran nach Aussen. Von 
grösserer Ausdehnung nach Innen hin ist der belle blasse Saum, welcher in gleicher Weise von feinen blassen Zellfortsätzen gebildet wird, welche nach innen gerichtet sind und sich in den Nervenbündeln verlieren. - Am Aequator besteht die distale Lamelle aus 8-9 Zellenlagen (bei 1,8 cm langen Kaninchenembryonen), aus 9-11 bei Rindsembryonen von 2-5,2 $\mathrm{cm}$ Länge; auch hier beobachtet man die Mitose mit tangentialer Teilungsebene in den Zellen der nahe der ausseren Oberfläche (Fig. 5a) befindlichen Lage, ebenso wie die Mitose mit radialer and tangentialer Theilungsebene in den Zellen der medianen Lagen (Fig. 5 b). Hier zeigen sich die Nervenfibrillen in dürftiger Entwicklung.

Im vorderen Theile besteht die distale Lamelle, von hinten nach vorn abnehmend, aus 5 Lagen der beschriebenen Zellen (bei $1,8 \mathrm{~cm}$ langen Kaninchenembryonen), aus 5 bis 3 Lagen bei 2-5,2 $\mathrm{cm}$ langen Rindsembryonen, mit Kerntheilungen in radialer und tangentialer Richtung. Am vorderen Ende der Ciliarportion bekommen die Zellen cylindrische Gestalt und sind zuletzt in der eigentlichen Ciliarportion auf eine Lage cylindrischer Zellen reducirt.

In einem weiteren Entwicklungsstadium zeigt sich die distale Lamelle aus zwei Zonen von Zellen zusammengesetzt, einer äusseren dunkeln und einer inneren hellen Zone $(2,7 \mathrm{~cm}$ langer Kaninchenembryo und $\mathbf{3}$ Wochen alter Meersehweinchenembry0).

Die dunkle äussere Zone wird am hinteren Pole von 7-12 Zellenlagen beim $2,7 \mathrm{~cm}$ langen Kaninchenembryo und von 15 Lagen beim 3 wöchentlichen Meerschweinchenembryo gebildet. Diese Zellen haben einen länglich ovalen und nur an der äusseren Oberfläche runden oder leicht ovalen Kern, zeigen zahlreiche Karyokinesen mit radialer oder auch tangentialer Theilungsebene in der oberflächlichen Schichte, aber auch in den Zellen, welche die mehr nach innen liegenden Schichten bilden (Fig. 6, A f); das Reticulum der länglich ovalen Kerne dieser Zone ist 
intensiv gefärbt, ebenso auch, aber etwas weniger, der Kernsaft; in den runden oder leicht ovalen Kernen ist nur das Reticulum gefärbt, dagegen der Kerninhalt beinahe immer farblos. - Am Aequator zeigt diese Zone dieselben Zellen mit der Karyokinesis in denselben Lagen, ist jedoch aus 6-9 Zellenlagen (beim $2,7 \mathrm{~cm}$ langen Kaninchenembryo) oder aus 9-10 Lagen (beim 3 wöchentlichen Meerschweinchenembryo) zusammengesetzt. - In der Ciliarportion bilden die Zellen mit ovalem Kern und von rein cylindrischer Gestalt ihre ganze Dicke; dieser Theil wird gebildet (von hinten nach vorn abnehmend) aus 3-2 Lagen (beim Kaninchenembryo von 2,7 cm Länge) oder aus 5-2 Lagen (beim 3 wöchentlichen Meerschweinchenembryo).

Das rordere Ende dieser pars ciliaris besteht aus einer Lage cylindrischer Zellen, welche Karyokinese mit radialer Theiling zeigen.

Die helle Innenzone der distalen Lamelle ist am hinteren Pole zusammengesetzt aus Zellen mit rund-ovalem Kern (Fig. 6, A g) der grösser ist als der Kern der Zellen der dunkeln Aussenzone, mit gefürbtem Reticulum, farblosem Kernsaft, aber intensiv gefärbtem Kernkörperchen; diese Zellen liegen weit auseinander und bilden 4-5 Lagen (beim 2,7 cm langen Kaninchenembryo und 3 wöchentlichen Meerschweinchenembryo). Am Aequator bilden diese Zellen 3-4 Lagen und man findet hier die Karyokinese mit tangentialer Theilungsebene. An der inneren Seite der Zellen dieser Zone bemerkt man feine blasse Zellfortsätze und die Fibrillen der Schicht der Nervenbündel.

Bei mensehlichen Embryonen ist der Entwicklungsprocess der distalen Lamelle, wie sie jetzt für andere Säugethiere beschrieben wurde, weiter vorgeschritten. Die distale Membran besteht bei 3,8 und $7 \mathrm{~cm}$ langen menschlichen Embryonen an ihrem hinteren Pole und am Aequator aus zwei Zonen: die dunkle Aussenzone ist zusammengesetzt aus Zellen mit sehr länglich-ovalem Kerne; doch erscheint 
dieser Kern in einer gewissen Anzahl von Zellen der äusseren Oberflächenlage rund oder wenig oval; diese Zone wird von 5-6 Lagen von Zellen mit intensiv gefärbtem Reticultum und schwach gefürbtem Kernsaft gebildet; in den Zellen der äusseren Oberfläche finden sich zahlreiche Kerntheilungen (Fig. $10 \mathrm{~A} . \mathrm{b}$ ) mit tangentialer und radialer Theilungsebene; doch beobachtet man die Mitose auch in den Zellen der medianen Lagen dieser Zone $(7 \mathrm{~cm}$ langer menschlicher Embryo). - Die Ciliarportion ist beim $3,8 \mathrm{~cm}$ langen menschlichen Embryo, (von hinten nach vorn abnehmend) aus 3-2 Lagen von Zellen mit länglich ovalem Kern, wie sie in der äusseren Zone der anderen Regionen der distalen Lamelle besehrieben wurden, zusammengesetzt, während sie beim $7 \mathrm{~cm}$ langen menschlichen Embryo in ihrem mehr nach vorn liegenden Theil aus cylindrischen Zellen gebildet wird. Hier beobachtet man die Kerntheilung in tangentialer Richtung. - Die helle Innenzone wird sowohl beim $3,8 \mathrm{~cm}$ langen als beim $7 \mathrm{~cm}$ langen menschlichen Embryo aus 5-6 Lagen von Zellen mit oralem oder rundem Kern, mit gefärbtem Reticulum und farblosem Kerninhalt (Fig. $10 \mathrm{~A} \mathrm{c}$ ) gebildet und zwar sowohl am hinteren Pol als am Aequator. - Nach Innen zu von diesen Zellen befinden sich die Fibrillenbündel des Nervus opticus, zwischen welchen man Zellen mit Kernen bemerkt, ähnlich denjenigen der Zellen der hellen Innenzone. Diese Zellen sind meistens parallel der Richtung der Nervenfibrillen gelagert; andere Zellen liegen nahe am vorderen Rande der Nervenbündel, wo sich auch die Fusspunkte der Müller'sehen Radialfasern bemerkbar machen.

In einer folgenden Periode der Entwieklung der Retina zeigt im Grossen und Ganzen die dunkle Aussenzone sowohl am hinteren Pole als am Aequator ein ähnliches Verhalten, wie es in der vorhergehenden Entwickelungsperiode besehrieben wurde; indess bemerkt man, dass an die Zellen ihrer inneren Grenze (Fig. 7a) ein heller farbloser Raum 
angrenzt, der aus feinen Zellenfäden gebildet wird (Fig. $7 \mathrm{~b}$ ). Derselbe ist die Anlage der inneren reticulären Schichte; in demselben bemerkt man Zellen mit runden und ovalen Kernen (Fig. 7 e) mit gefürbtem Reticulum, aber stärker gefärbtem Nucleolus, während der Kernsaft farblos ist. Diese Zellen bilden $2-4$ Lagen (beim $4,3 \mathrm{~cm}$ langen Kaninchenembryo) oder $6-8$ Lagen (beim $6,2 \mathrm{~cm}$ langen Rindsembryo); in einigen dieser Zellen, welche in der Nähe der Nerventibrillen liegen, bemerkt man Karyokinese (Fig. 7d). Von den Zellen der distalen Lamelle gehen Fortsätze aus, von denen die nach innen gerichteten bis zu den Opticasfasern vordringen und sich mit ihnen verbinden; zwischen letzteren beobachtet man auch in Kerntheilung (Fig. $7 \mathrm{e}$ ) befindliche Zellen und Andeutungen der Basen der Mullersehen Radialfasern. In der Pars ciliaris besteht die distale Lamelle (von hinten nach vorn abnehmend) aus 4-1 Lagen eylindrischer Zellen mit ovalen oder runden Kernen und intensiv gefürbtem Reticulum (beim 4,3 cm langen Kaninchenembryo), während sie beim $6,2 \mathrm{~cm}$ langen Rindsembryo 4-1 Lagen von Zellen mit länglich-ovalem Kern bildet.

Im weiteren Fortschritt der Entwicklung der distalen Lamelle (bei $5,7 \mathrm{~cm}$ langen Kaninchenembryonen, 7,5-8 $\mathrm{cm}$ langen Rindsembryonen, $16,3 \mathrm{~cm}$ langen Hundeembryonen) bemerkt man am hinteren Pole und am Aequator, dass die Zellen der dunklen Aussenzone, welche an ihrer inneren Grenze liegen, wichtige Veränderungen eingehen; sie zeigen einen Kern nicht mehr von länglich ovaler Form, mit lebhaft gefärbtem Reticulum und auch etwas gefärbtem Kernsaft, sondern ron mehr breit ovaler Gestalt (Fig. 8 a) mit gefärbtem Reticulum und Kernkörperchen und mit farblosem Kerninhalt; diese Zellen sieht man allmählich übergehen in die des Stratum reticulare internum, welche eine weitere Entwickelungsstufe jener sind. Die Zellen der inneren hellen Zone (Fig. $8 \mathrm{c}$ ) zeigen Kerne von mehr breit ovaler, unregelmässig runder, manch- 
mal aber anch länglicher Gestalt mit gefärbtem Reticulum und Kernkörperchen mit blassem Kernsaft, diese Zellen zeigen deutlich einen Fortsatz nach innen und aussen, bilden 2-3 Schichten (bei 5,7 cm langen Embryonen von Kaninchen und $16,3 \mathrm{~cm}$ langen Hundeembryonen) oder 3 bis 7 Sehichten (bei 7,5-8 cm langen Embryonen vom Rinde); in diesen Zellen bemerkt man die Kerntheilung auch in der Nähe der Nervenfibrillen (Fig. $8 \mathrm{~d}$ ).

Die Pars ciliaris retinae der distalen Lamelle zeigt in dieser Epoche dasselbe Entwickelungsstadium, wie es in der vorhergehenden Periode beschrieben wurde.

Bei Embryonen der folgenden Epoche werden die Charaktere der Zellen der inneren hellen Zone deutlicher. In der That findet man bei $8,5 \mathrm{~cm}$ langen Kaninchenembryonen, beim ausgetragenen Kaninchenfötus, bei $13,3 \mathrm{~cm}$ langen Rindsembryonen die Membrana limitans externa fertig gebildet. Die dunkle Aussenzone hat dieselbe Structur wie in der vorhergehenden Periode und die Zellen der M. limitans interna zeigen Kerne von einem grösseren Oval mit gefärbtem Reticulum und Kernkörperchen und mit vollständig ungefärbtem Kernsaft. Das Stratum reticulare internum ist deutlicher. Im Innern desselben bemerkt man zwei Arten von Zellen; die einen mit grossem runden oder leicht ovalen Kern mit gefärbtem Reticulum, intensiv gefärbten Kernkörperchen und farblosem Kernsaft bilden am hinteren Pole 2 Lagen, am Aequator nur eine Lage und sind in Abständen von einander gelagert. Zwischen den beschriebenen Zellen finden sich die anderen in 2-3 Lagen angeordnet mit ovalen Kernen, welche kleiner sind als die der ebenerwähnten Zellen mit intensiv gefärbten Reticulum und etwas gefärbtem Kernsaft; diese letzteren Zellen findet man auch in der Nervenfaserschichte und bei den Müller'schen Radialfasern. - Die Pars ciliaris der distalen Lamelle zeigt denselben Entwicklungszustand wie in der vorhergehenden Periode, nur die Cylinderzellen, welche sie an 
ihrem vorderen Ende bilden, zeigen einen Kern mit intensiv gefärbtem Reticulum. Bei allen Embryonen dieser Epoche bemerkt man den Kerntheilungsprocess in den Zellen der äusseren Oberflächenschicht der dunklen Aussenzone.

Bei anderen Săugethierembryonen hat die Histogenese der eigentlichen Retina eine beträchtliche Fntwicklung erlangt. - Beim menschlichen Fötus von $21,5 \mathrm{~cm}$ Lănge zeigt die eigentliche Retina von aussen angefangen, folgenden Entwicklungszustand. Die Membrana limitans externa (Fig. 11, A a) ist in Form einer leicht gewellten feinen Linie vorhanden, auf welche die Stäbchen und Zapfen anstossen oder welche diese auch durchsetzen.

Die Entwicklung der Stäbchen vollzieht sich in folgender Weise. Die Kerne der an die Membrana limitans externa angrenzenden Zellen verdünnen sich und nehmen die Gestalt eines feinen kurzen Stäbchens an (Fig. $11 \mathrm{~A} \mathrm{~b}$ ), neben welchem sich gewohnlich eine Zelle mit kleinem ovalem Kerne ohne Fortsatz nach Innen befindet. Sowohl das Stäbchen als auch der ovale Kern der eben erwähnten Zelle haben ein intensiv gefärbtes Reticulum und einen ebensolchen Kerninhalt; daher kommt manchmal bei gut gelungener Färbung eine Contrastwirkung zu Stande zwisehen der Intensität der Färbung dieser Elemente und der blasseren Färbung der in Entwicklung begriffenen Zapfen und der Kerne, aus welchen diese hervorgehen. Die in Entwicklung begriffenen Stäbchen haben an ihrem vorderen Ende einen kleinen Anhang blasser protoplasmatischer Zellsubstanz und an ihrem inneren Ende einen blassen Fortsatz, der sich in ein feinstes Netzwerk hineinbegiebt, wo auch er sich in Fäden theilt. Dieses Netzwerk repräsentirt die Anlage des Stratum reticulare externum. Manchmal bemerkt man einen ovalen Kern mit intensiv gefärbtem Netzwerk und einem leicht gefärbten Kernsaft über dem innern Fortsatz der Stäbchen liegen. Bei fortschreitender Entwicklung erreichen die Stäbchen die äussere 
Grenzmembran und durehsetzen dieselbe, zeigen aber auch dann noch den beschriebenen Protoplasmaanhang (Fig. 11 A c). Die Zapfen sieht man sich aus ovalen Zellkernen entwickeln, welche dicker und etwas länger werden (Fig. $11 \mathrm{Ad}$ ) und deren Inhalt leicht gefärbt oder auch farblos ist, während dagegen das Reticulum und auch das Kernkörperchen gefärbt ist; an dem innern Ende der Kerne der Zellen, in welchen sich der Zapfen entwickelt, findet sich ebenfalls ein blasser Fortsatz, der sich in das oben erwähnte Netzwerk hineinbegiebt und in verschiedene Fäden auflöst. Die ovalen Kerne, aus welchen sich die Zapfen entwickeln, bilden auch an ihrem dickeren Ende, welches das äussere ist, einen Protoplasmaanhang. Indem sie allmählich mehr oval und grösser werden, gelangen sie an die Membrana limitans externa, durchsetzen dieselbe, wobei sie zum Theil ihre Gestalt beibehalten; daher sind sie, wenn sie vollständig über die Membrana limitans nach Aussen hervorragen, mit dieser mittelst eines Stiels verbunden, wodurch die Zapfen in dieser Gestalt Aehnlichkeit bekommen mit umgekehrten Flaschen oder mit gestielten ovalen Bläschen; dieser Stiel ist ihr innerer Fortsatz, der sich in das Netzwerk des Stratum reticulare externum hineinbegiebt. Wenn die Zapfen einmal diese Entwicklungsstufe erreicht haben, zeigen sie den Protoplasmaanhang an ihrer äusseren Oberfläche nicht mehr. Zwischen den Elementen, welche die Zapfen und Stäbchen bilden und dem Orte, wo das Stratum reticulare externum angelegt wird, existirt eine blasse Strecke, welche von den inneren Fortsätzen der Stäbchen und Zapfen durehsetzt und auch hie und da von den spärlichen beschriebenen Zellen eingenommen wird, welche sich meist neben den erwähnten in Entwicklung begriffenen Elementen befinden. Einwärts von der Stelle, an welcher das Reticulum (Stratum reticulare externum) aufgetreten ist, befindet sich eine Lage von Zellen mit rundem oder leicht ovalem Kern mit intensiv gefärbtem Reticulum und Kernsaft (Fig. 11 A f). Diese 
Zellen bemerkt man mitten unter andern in dieser ganzen Zone.

Unterhalb der Linie der erwähnten Elemente bemerkt man drei Lagen von Zellen mit ovalem oder rundem Kern (Fig. $11 \mathrm{~A} \mathrm{~g}$ ), mit gefärbtem Reticulum und Kernkörperchen, aber mit ungefärbtem Kernsaft; unmittelbar unterbalb der dritten Lage dieser Zellen bemerkt man eine Lage bipolarer Zellen, auch einzelne multipolare und spindelförmige Zellen (Fig. $11 \mathrm{~A} \mathrm{~h}$ ) mit Fortsätzen, welche zumeist nach Aussen und Innen gerichtet sind und sich intensiv färben.

Nach Innen von diesen Zellen haben wir wieder 3 Lagen von Zellen mit grossem Kern (Fig. $11 \mathrm{~A} \mathrm{~g}$ ), welche sich ebenso verhalten wie diejenigen, welche nach Aussen von den bipolaren Zellen liegen und beschrieben wurden. Die innere reticuläre Schicht wird von einem feinsten Netz von Zelleufäden gebildet und enthält hier und da Zellen mit ovalem oder grossem rundem Kern (Fig. $11 \mathrm{~A} \mathrm{i)} \mathrm{mit} \mathrm{ge-}$ färbtem Reticulum und ungefärbtem Kerninhalt; daneben lassen sich andere Zellen mit kleinem Kern, mit gefärbtem Reticulum und mit gefürbtem Kerninhalt finden. Nach Innen von dem Stratum reticulare internum bemerkt man Zellen mit grossen runden Kernen mit gefärbtem Reticulum und Kernkörperchen mit ungefärbtem Kerninhalt (Fig.11Ak), welche in zwei Lagen angeordnet und von einander entfernt liegen; mitten unter ihnen finden sich andere Zellen mit kleinerem Kern (Fig. 11 A I) mit intensiv gefärbtem Reticulum und Kerninhalt; sie bilden vier oder fünf Lagen zwischen denen der grossen Zellen, die in dieser Zone beschrieben wurden, in welcher man auch die Müller'schen Radialfasern bis zu ihrem vorderen Ende bemerken kann. (Fig. 11 A n). Bei diesen bemerkt man auch die Nervenfibrillen (Fig. $11 \mathrm{~A} \mathrm{~m}$ ).

Bei dem $7 \mathrm{~cm}$ langen Meerschweinchenfötus sind die Stäbchen und Zapfen auf dem Wege, sich aus den Zellen der âusseren Oberflächenschicht zu entwickeln, wie es eben 
beim menschlichen Fötus beschrieben wurde; alle anderen Lagen der Retina sind jedoch bereits entwickelt.

Beim neugebornen Kaninchen zeigt die distale Lamelle in der dunkeln Aussenzone die Membrana limitans externa und nach Innen von derselben sowohl am hinteren Pole als am Aequator 10-11 Lagen von Zellen mit ovalem Kern, mit stark gefärbtem Retienlum und auch etwas gefärbtem Kerninhalt. Doch finden sich in der äussern Oberflächenschicht auch Zellen mit runden Kernen. In den Zellen der äusseren Oberflächenschicht findet Kerntheilung statt.

An der inneren Grenze dieser Zone haben die Zellen einen grossen ovalen Kern mit gefürbtem Reticulum, aber mit lebhaft gefärbten Kernkörperchen und mit ungefürbtem Kerninhalt (Fig. 9 b). Nach Innen von dem Stratum reticulare internum giebt es zwei Lagen von Zellen, von denen die einen einen ovalen, runden (Fig. 9d), grossen Kern mit gefärbtem Reticulum, intensiv gefärbtem Nucleolus und nngefärbtem Kernsaft und mit einem nach aussen oder nach innen gerichteten sichtbaren Fortsatz haben: es sind dies vollständig entwickelte Ganglienzellen. Die anderen Zellen (Fig. 9c) haben einen runden oder ovalen Kern von geringerem Umfang mit intensiv gefärbtem Reticulum und Kernkörperchen und ebenfalls gefärbtem Kernsaft. Die Lagen dieser Zellen sind nach innen von der der Nervenfibrillen begrenzt, zwischen denen sich Zellen mit oralem Kern bemerkbar machen, deren grösste Achse in der Richtung der Nervenfasern verläuft. - Der Ciliartheil der distalen Lamelle ist von hinten nach vorn gezählt aus 3-2 Lagen cylindrischer Zellen mit länglich ovalem Kern gebildet, welche sich weiter nach vorn an den Ciliarfortsätzen auf eine einzige Lage ebenfalls cylindrischer Zellen reduciren.

Beim menschlichen Neugeborenen and bei dem des Meersehweinchens sind die Schichten der Retina vollständig entwickelt, die Stäbchen und Zapfen mit inbegriffen.

Beim 5 Tage alten Kaninchen ist noch keine Spur 
von Stäbchen und Zapfen vorhanden. Eine blasse helle Linie theilt die dunkle Aussenzone in zwei Lagen von Zellen. In den Zellen der nun zur äusseren gewordenen Zone bemerkt man karyokinetische Figuren mit tangentialer Theilungsebene bei den Zellen der äusseren Oberfläche und mit radialer Theilungsebene bei denjenigen, welche in der Nähe der erwähnten blassen Linie liegen. Die übrige Retina verhält sich genau, wie es beim neugeborenen Kaninchen beschrieben worden ist und die Membrana limitans externa ist fertig gebildet.

Beim Kaninchen von 15 Tagen sind die Stäbchen und Zapfen entwickelt. Man bemerkt in den Kernen der Stäbchen, wie Henle fand und M. Schultze, Merkel, G. Wagner, W. Krause, Schwalbe and Dennisenko bestätigten, dass sie aus 2 transversal gestellten Scheiben bestehen, welche intensiv gefärbt sind und an den Polen des Kernes liegen; manchmal bemerkt man auch noch eine dritte Scheibe, welche transversal zwischen den beiden anderen liegt. Diese Thatsache erklärt Flemming*) so, dass in den Kernen der Stäbchen sich 2 Substanzen von verschiedenem Lichtbrechungsvermögen befinden, von welchen die eine bei gewissen Färbemethoden sich schnell färbt und die ebenerwähnten gefärbten Scheiben darstellt, während die andere farblos bleibt.

Das Stratum reticulare externum zeigt an der Innenseite Zellen mit ovalen Kernen, welehe mit der grössten Achse der transversalen Richtung eines Theiles der Zellenfäden, welche denselben bilden, parallel gerichtet sind.

Die Kerne dieser Zellen haben ein gefürbtes Reticulum und Kernkörperehen, während der Kernsaft farblos ist.

Die innere Körnerschicht zeigt 2-3 Lagen von Nervenzellen mit ovalen und runden Kernen, mit gefärbtem Reticulum und Kernkörperchen und farblosem Kerninhalt und

*) Zellsubstanz, Kern- und Zelltheilung. 1882, p. 115. 
mitten zwischen denselben eine Sehicht bipolarer intensiv gefărbter Zellen.

Die Ganglienzellen bilden eine einzige Lage. Diese Zellen haben einen grossen Kern mit gefärbtem Retieulum und Kernkörperchen und ungefärbtem Kerninhalt und einem sichtbaren Fortsatz nach innen. Zwischen diesen Zellen bemerkt man andere mit kleineren Kernen, welche ein intensiv gefärbtes Reticulum und einen auch etwas gefärbten Kerninhalt besitzen.

Die anderen Schichten der Retina zeigen sich deutlicher entwickelt als bei Kaninchen der vorhergehenden Epoche. Der Process der Karyokinese hat aufgehört.

Beim Kaninchen von 30 Tagen haben die verschiedenen Schichten der Retina ihre Entwicklung erreicht und zeigt sich ihre Structur deutlich.

Bei Kaninchen von 40-42 Tagen hat die Retina vollständig die Structur wie im ausgewachsenen Zustande.

\section{Histogenese des Epithels der Retina.}

Nach der Bildung der secundären Augenblase beginnt die proximale Lamelle dünner zu werden, während die distale in die Dicke zu wachsen anfängt. In der That besteht sie bei Rindsembryonen von $18 \mathrm{~mm}$ Länge am hinteren Pole aus einer, zwei, auch drei Lagen cubischer Zellen (Fig. 2, A d) mit (von der Seite gesehen) ovalem, in seinem Innern nach der distalen Lamelle gerichteten Theil pigmentirten Kern. Im Frontalschnitt erscheinen diese Zellen in verschiedener Gestalt, rundlich oder polygonal und ist ihr Kern oval (Fig. 2, B i). Das Pigment erscheint in Form kleiner Körnchen, umgrenzt die Zellensubstanz, während der Kern vollständig frei davon bleibt; in den von der Seite gesehenen Zellen nimmt das Pigment ihren inneren Theil ein und erscheint alsdann in Gestalt kleiner Stäbchen. Diese Zellen der proximalen Lamelle setzen sich fort in diejenigen, welche zum grössten Theil das Stützgewebe des 
Nervus opticus bilden (Fig. 2, A e) und hier sowie in der Nähe dieses Punktes bilden die cubischen Zellen 2 bis 3 Lagen und sind frei von Pigment.

Am hinteren Pole bemerkt man zwischen diesen Zellen der proximalen Lamelle und den Zellen der äusseren Oberfläche der distalen Lamelle andere Zellenelemente (Fig. 2, A f), welche wahrscheinlich vom Mesoderma abstammen und welche ein Bindemittel zwisehen der distalen Lamelle und dem Rudiment des Nervus opticus sind. Am Aequator wird die proximale Lamelle von den am hinteren Pole beschriebenen Zellen gebildet, doch bilden dieselben nur eine einzige Lage. - In ihrem vorderen Theil vermehren sich die Zellen der proximalen Lamelle auf 3 Schichten; sie haben einen länglich ovalen Kern; diese Schichten reduciren sich weiter nach vorn auf zwei und zwar bis zu der Stelle, wo die proximale an die distale Lamelle angrenzt. Das Pigment nimmt in dieser Region nicht nur den inneren Theil der Zellen der weiter nach innen liegenden Schicht ein, sondern es findet sich zuweilen auch in den Zellen der äusseren Schicht.

Im weiteren Verlauf der Entwicklung (bei Kaninchenembryonen von $1,8 \mathrm{~cm}$ und bei Rindsembryonen von $2-5,2 \mathrm{~cm}$ ) besteht die proximale Lamelle an ihrem hinteren Pole und am Aequator (in der Seitenansicht) aus einer Lage cubischer Zellen (Fig. $3 \mathrm{~B} \mathrm{f}$ ), welche besonders in ibrem inneren Drittel stark pigmentirt sind und einen pigmentfreien Kern besitzen. Diese Zellen haben, von vorn gesehen, eine polygonale Gestalt (Fig. $3 \mathrm{Bg}$ ). Das Pigment liegt in der Peripherie der Zelle und lässt den Kern frei. Die vordere Portion der proximalen Lamelle ist ähnlich so, wie sie in der vorhergehenden Periode beobachtet wurde, nur beim Rindsembryo von $5,2 \mathrm{~cm}$ ersoheint die Pigmentirung entsprechend der Entwicklung des Ciliarkörpers viel intensiver. - Das Tapetum besteht bei Kaninchenembryonen von $2,7 \mathrm{~cm}$ und bei 6 wöchentlichen Meerschweinchen- 
embryonen am hinteren Pole und am Aequator aus einer Lage (in Seitenansicht) cubischer Zellen (Fig. $6 \mathrm{~A} \mathrm{~h}$ ), welche in Karyokinesis begriffene Kerne zeigen (Fig. $6 \mathrm{~B}$ e), was man auch in den (in Seitenansicht) cylindrischen Zellen des Ciliartheils beobachtet, wo die proximale Lamelle aus ẹiner einzigen Lage dieser Zellen zusammengesetzt ist. Die Zellen des Tapetum zeigen in der Ansicht von vorne an ihrem hintern Pol und am Aequator (Fig. $6 \mathrm{~B} \mathrm{k}$ ) eine polygonale Gestalt, ihre Conturen sind grösstentheils verdeckt von dem Pigment, das in die Zellsubstanz bis an den Kern heran eingedrungen ist, dessen Umriss daher auch nicht deutlich erscheint. Bei menschlichen Embryonen von $3,8 \mathrm{~cm}$ ist das Tapetum gleichfalls aus cubischen Zellen gebildet (Fig. $10 \mathrm{Ag}$ ) (Seitenansicht), welche einen ovalen Kern besitzen; diese Zellen zeigen in der Ansicht von vorn eine polygonale Gestalt (Fig. $10 \mathrm{~B} \mathrm{~h}$ ) mit leicht ovalem Kern. In denselben findet man das Pigment in der Peripherie angehäuft, doch findet man auch welches in dem Kern, wenn auch in geringerer Menge als in der Zellsubstanz. Am. Aequator und in dem vorderen Theil verhalt sich das Tapetum wie es bei den andern Säugethieren vorhin beschrieben worden ist. - Bei menschlichen Embryonen von $7 \mathrm{~cm}$ bilden die Zellen des Retinaepithels in der Seitenansicht einen schmalen Streifen kleiner Platten; in der Ansicht von vorn erscheinen sie in hexagonaler Gestalt Der Kern ist fast vollständig pigmentfrei und liegt auf einer Seite der Zelle, wesshalb das Pigment in der Zellsubstanz in einer Weise angehäuft liegt, dass es zwischen sich und dem Kern einen fast vollständig freien Hof lässt. - Bei weiter entwickelten Embryonen (Kaninchen von 4,3 und $5,7 \mathrm{~cm}$, Rind von 6-8 cm) erscheinen die Pigmentzellen des Tapetum wie bei Embryonen der vorhergehenden Epoche; nur bei den eben erwähnten Kaninchenembryonen bemerkt man an den Zellen in der Seitenansicht, dass ihre langen Protoplasmafortsätze stark pigmentirt und nach der distalen 
Lamelle hin gerichtet sind, an deren äusserer Grenze man zuweilen Spuren von Pigment erblickt. - Beim menschlichen Embryo von 21,5 cm erscheinen die Zellen des Tapetum in der Seitenansicht als ein dünner Streif von Plättchen (Fig. $11 \mathrm{~A} 0$ ), in der Ansicht von vorn zeigen sie hexagonale oder polygonale (Fig. $11 \mathrm{~B}$ p) Gestalt mit meist aus dem Centrum nach einer Seite hin rerschobenem, mit einem halbmondförmigen Pigmenthof umgebenem Kern. Wenn manchmal der Kern in der centralen Zone der Zelle sich befindet, dann ist das Pigment in Form eines Rings um den Kern herum vertheilt.

Bei anderen Säugethieren (Rindsembryo von $13,3 \mathrm{~cm}$, Kaninchenembryo von 8,5 und Meerschweinchenfötus von $7 \mathrm{~cm}$ ) haben die Zellen des Tapetum die Charaktere erlangt, welche man an den Thieren nach der Geburt findet. Man beobachtet daher, dass beim neugebornen Kaninchen (Fig. 9 i) die Zellen des Retinaepithels in der Seitenansicht gerade wie beim neugebornen Menschen und Meerschweinchen sich in der Gestalt feiner Platten präsentiren, während diese Zellen in der Ansicht von vorn eine deutlich hexagonale Gestalt mit ovalem Kern zeigen und das Pigment die Zellsubstanz und auch den Kern occupirt hat. Bei Kaninchen von 7-15-30-40-42 Tagen zeigen die Zellen des Retinaepithels sich vollständig entwickelt, wie es für das neugeborne Kaninchen besehrieben wurde.

Aus meinen Auseinandersetzungen über die Histogenese der Retina der Säugethiere ergiebt sich folgendes: Bei der Histogenese der Retina geschieht die Vermehrung ihrer Elemente durch den Process der Karyokinese, welcher bei einigen Säugethieren (Kaninchen) bis zum 7. Tag nach der Geburt anhält. Den Vorgang der Mitose bemerkt man nicht nur in den Zellen der äusseren Oberflächenschicht der distalen Lamelle, sondern auch in den anderen Schichten dieser Lamelle, doch sind in der äusseren Oberflächenv. Graefe's Arehiv für Ophthalmologie, XXXIV. 2. 
schicht die Mitosen zahlreicher als in anderen Schichten. In allen Regionen der distalen Lamelle, sowohl am hinteren Pol als am Aequator, als an dem Ciliartheil bemerikt man die Karyolinese in den Zellen der versehiedenen Schichten. Die Richtung der Theilungsebene ist bei der Histogenese der Retina meistens eine tangentiale, weniger häufig eine radiale und noch viel seltener eine diagonale. Die Karyokinese hört auf, sobald die Stäbchen ihren Entwickelungsprocess beginnen.

Mit Rücksicht auf die histologischen Studien von Flemming und Strasburger und die chemischen von Zacharias, Plösz und Kossel über die Zelle erhielt ich folgende Resultate. Die erste Verschiedenheit in der Gestalt und dem Farbenton der Zellkerne der proximalen und distalen Lamelle macht sich bemerklich, wenn sich die secundäre Augenblase bildet. Kaum hat sich die distale Membran verdickt, so zeigt sie Zellen mit intensiv gefärbtem Kern and Reticulum, während der Kernsaft nur wenig gefärbt ist. Bald darauf beginnt eine Umgestaltung der Zellen, welche an dem inneren Rande der distalen Lamelle liegen. Die Kerne der dort gelegenen Zellen werden oval und grösser, ihr Reticulum und Kernkörperchen wird gefärbt. Nach Bildung des Stratum reticulare internum entstehen aus den vorher erwähnten Zellen zwei Arten, die einen haben einen kleineren Kern mit intensiv gefärbtem Reticulum und auch etwas gefärbtem Kerninhalt, die anderen Zellen haben einen grossen Kern mit gefärbtem Reticulum und Kernkörperchen, aber ungefärbtem Inhalt. Diese letzteren Zellen zeigen bei ihrer Weiterentwicklung Fortsätze und bilden die Ganglienzellen der Retina.

Aus der äusseren Zellenschicht der distalen Lamelle stammen die Stäbchen und Zapfen (beim Kaninchen zwischen dem 7. und 15. Tag nach der Geburt und nicht schon beim neugebornen Kaninehen, wie Koganei glaubt und in Fig. 3 seiner Arbeit abbildet). Desshalb gebe ich nach vielen 
Präparaten in Figur 10 eine Darstellung der Retina des neugebornen Kaninchens. Was Koganei als Stäbchen und Zapfen bezeichnet, findet sich bereits beim Kaninchenfotus von $8,5 \mathrm{~cm}$, ist aber nichts anderes als die äussersten Enden der Zellenfortsätze der distalen Lamelle. Dagegen entwickeln sich die Stäbchen und Zapfen bei Säugethieren (menschlicher Fötus von $21,5 \mathrm{~cm}$ und Meerschweinchenfötus von $7 \mathrm{~cm}$ ) aus den Kernen der äusseren Oberflächenschicht durch den oben von mir beschriebenen Vorgang (Fig. 11 A bcde). Am inneren Ende haben sie einen blassen Fortsatz, welcher sich in Fäden theilt und sich in das sehr feine Netzwerk des Stratum reticulare externum hineinbegiebt.

Die Stäbchen und Zapfen zeigen während ihrer Entwicklung einen leichten Unterschied in der Intensität ihrer Färbung. Der übrige Theil der Entwicklung der Retina, welchen Koganei dem neugebornen Kaninchen zuschreibt, das heisst die Bildung der Körnerschichten mit dem Erscheinen des Stratum reticulare externum existirt in Wirklichkeit beim neugeborenen Kaninchen nicht, man findet vielmehr diese Verhältnisse zuerst beim Kaninchen von 7 Tagen.

Beim Kaninchen von 15 Tagen kommen an den Kernen der Stäbchen durch die Färbung die queren Scheiben zur Beobachtung, welche von Henle entdeckt und von Flemming auf das Vorhandensein zweier Substanzen von verschiedenem Brechungsindex zurückgeführt wurden, von welchen die eine sich mit gewissen Färbemitteln färben lässt, die andere aber farblos bleibt.

Die innere und äussere reticuläre Schicht entwickeln sich so, wie es Babuchin, Ogneff und Kölliker beschrieben haben.

Das Tapetum nigrum ist das Entwicklungsproduct des äusseren Blattes der secundären Augenblase. Kurz nach seiner Bildung besteht, es aus (von der Seite gesehen) 
cubischen Zellen, welche sich durch Karyokinese vermehren und zwar sowohl am hinteren Pol als am Aequator; in dem vorderen Theil besteht es aus cylindrischen Zellen, welche ebenfalls Mitose zeigen. Das Pigment befindet sich im innern Theil dieser cubisehen Zellen and stärker angehäuft in den cylindrisehen Zellen des vorderen Theils, wo es eine oder zwei Lagen derselben einnimmt. In der Ansicht von vorn erscheinen diese cubischen Zellen rund oder unregelmässig polygonal und das Pigment hört an der Grenze der Zellsubstanz auf. Die cylindrischen Zellen würden nach der Theorie von Hennum aus den eubischen in Folge von Druckwirkung von Seiten der letzteren entstehen; aus derselben Ursache würden die letzteren auch die platten Zellen entstehen lassen, welche, von vorn gesehen, hexagonal aussehen. Das Pigment nimmt die Zellsubstanz ein, dringt zuweilen aber auch bis in den Kern ein.

Bei Kaninchenembryonen von 2,7-4,3-5,7 cm Länge bemerkt man, dass die Zellen des Retinaepithels stark pigmentirte Fortsätze gegen die distale Lamelle zu ausschicken, auf deren Oberfläche man Pigment abgelagert findet — und dies Verhalten besteht zu einer Zeit, wo die Nervenzellen der Retina noch in dem Process der Entwicklung begriffen sind and die Stäbchen und Zapfen noch nicht existiren.

\section{Histogenese des Nervus optieus.}

Nach der Bildung der secundären Augenblase setzt sich die proximale Lamelle in die Wände des Stiels fort, welche dann sich weiter in die Wand der vorderen Hirnblase verlängern. Die Structur der Stielwand ist in diesem Stadium ähnlich der der proximalen und distalen Lamelle; sie lässt im Imnern einen Hohlraum. Im weiteren Verlauf der Entwicklung der secundären Augenblase geht der Stiel beträchtliche Veränderungen ein. Bei Rindsembryonen von $1,8 \mathrm{~cm}$ besteht er aus Zellen mit ovalem, etwas länglichem Kern inmitten eines sehr feinen Netzwerks; diese Zellen 
sind denen der proximalen Lamelle, deren Fortsetzung sie sind (Fig. $2 \mathrm{~A}$ e), ähnlich; ihre Kerne haben ein gefärbtes Reticulum und Kernkörperchen, während ihr Kernsaft farblos ist; sie liegen meistens mit ihrer grössten Achse in der Richtung der Achse des Stieles. Zwischen diesen Zellen verlaufen feine Nervenfäden (Fig, $2 \mathrm{Ag}$ ) in der ganzen Ausdehnung des Stieles; an den Seitenrăndern ist diese Anlage des Nerven von zarten wellenförmigen Falten des umgebenden Mesoderma begrenzt wie ron einer Anlage der Nervenscheide. Das den Nerven umgebende Mesoderma besteht aus Zellen mit länglich ovalem Kern, mit intensiv gefärbtem Reticulum and Kerninhalt und mit einer Richtung, welche gegen diejenige, in welcher die Zellen und die beschriebenen feinen Faden des Nerven verlaufen, senkrecht oder schräg steht; diese Mesodermazellen zeigen Karyokinese (Fig. $2 \mathrm{~A} \mathrm{~h}$ ). Zwischen dem ausseren Rande des hintern Pols der distalen Lamelle und dem Punkte, an welchem die proximale an der Bildung der Nervenanlage theilzunehmen beginnt, bemerkt man eine Anhäufung von wahrscheinlich dem Mesoderma zugehörigen Zellen, welche ein Bindeglied zwischen der distalen Lamelle und dem sich entwickelnden Nerven darstellen (Fig. 2 A f). - Im Verlauf der Weiterentwicklung besteht der Nervus opticus (beim Kaninchenembryo von $1,8 \mathrm{~cm}$, beim Rindsembryo von $2-5,2 \mathrm{~cm}$ und beim 3 wöchentlichen Meerschweinchenembryo) aus feinsten Nervenfibrillen und aus zahlreichen, gewöhnlich mit grossen Kernen, manchmal aber auch mit kleinen, runden oder länglich ovalen und zuweilen auch dreieckigen Kernen versehenen Zellen. Diese Zellen zeigen sich in der Gegend der Papille und hinten in der Linie, wo sich später die Lamina cribrosa befinden soll, mit der grössten Achse den Sehnervenfasern parallel gerichtet; die Zellen sind auch dort zahireich, wo die Ausbreitung des Nervus opticus beginnt; in den Kernen dieser Zellen an der Papille bemerkt man die Karyokinese (Fig $3 \mathrm{~A}$ b) mit meist gegen den Verlauf der 
Nervenfibrillen parallelen, manchmal aber auch schrägen Richtung; dagegen findet man in dem Niveau der Schicht, wo später die Lamina cribrosa entsteht, Zellen, welche senkrecht zur Richtung der Nervenfibrillen gestellt sind; sie haben länglich ovale, etwas kegelförmige oder spindelförmige Kerne (Fig. $3 \mathrm{~A} \mathrm{c}$ ) und zeigen eine Karyokinesis mit senkrecht zu den Nervenfibrillen gestellter Theilungs-Ebene (Fig. $3 \mathrm{~A} \mathrm{~d}$ ). Hinter dieser Stelle sind die Zellen sehr zahlreich, bilden keine regelmässige Streifen mehr und ihre Kerne sind rund, länglich oval und beinahe dreieckig mit der grössten Achse in der Richtung der Nervenfibrillen; unter ihnen finden sich Zellen, welche in Karyokinese begriffen sind mit parallel zur Richtung der Nervenfibrillen gestellter Theilungsebene. Alle diese Zellenelemente des Nervus opticus zeigen einen Kern mit leicht gefärbtem Inhalt, während das Reticulum und das Kernkörperchen intensiv gefärbt sind. In der Papille haben die Nervenfibrillen folgende Richtung: die mittleren Nervenbündel derselben erscheinen auf Längsschnitten vorn in der Form des freien Endes eines Pinsels (Fig. 3 A h), während die Nerrenfibrillen der Peripherie (Fig. 8 A i) sich nach vorn fortsetzen und wie der umkehrende Strahl eines Springbrunnens (wie sich Schwalbe ausdrückt) auseinanderbiegen, an der Innenseite der distalen Lamelle weiterlaufen und die Ausbreitung des Nerrus opticus bilden. In dem Mesoderma, welches den Nervas opticus umgiebt, finden sich viele Zellen mit ovalem oder rundem Kern mit intensiv gefärbtem Reticulum, welche zahlreiche Mitosen zeigen.

In weiteren Entwicklungsstadien (Fmbryo vom Kaninchen von $2,7 \mathrm{~cm}$, vom Meersehweinchen von 3 Wochen) sind die Fibrillen sowobl im Nerven als in der Papille deutlicher. Die zwischen diesen Fibrillen liegenden Zellen zeigen in Bezug auf Richtung und den Process der Karyokinese dasselbe Verhalten, wie in der zuletzt beschriebenen 
Entwicklungsperiode; in derselben Weise erscheinen im Gebiet der Papille die centralen Nervenbündel, welche in der Richtung von vorn nach hinten abgetheilt sind, wie das freie Ende eines Pinsels (Fig. $6 \mathrm{~A} \mathrm{a}$ ) und treten bestimmter hervor, während die Fibrillen der Peripherie weiter nach Forn verlaufen, um sich dann nach Aussen umzubiegen and so die Ausbreitung des Nerven zu bilden (Fig. 6 A c). Das Mesoderma, welches den Nervus opticus umgiebt, zeigt in dieser Epoche zahlreiche Mitosen. - Im Nervus opticus des menschlichen Embryo von $3,8 \mathrm{~cm}$ bemerkt man dasselbe Structurverhältniss wie in dem Stadium, welches eben für andere Säugethiere geschildert worden ist. Auch hier giebt es in Karyokinese begriffene Zellen, auch hier sieht man gleicher Weise in der Form eines Pinselendes hervortreten (Fig. $10 \mathrm{~A} \mathrm{~d}$ ), während die peripheren Nervenbündel zur Bildung der Nervenausbreitung des Nervus opticus sich strahlenformig auseinanderbiegen (Fig. $10 \mathrm{~A} \mathrm{e}$ ) und sich innerhalb der distalen Lamelle fortsetzen. Beim $7 \mathrm{~cm}$ langen menschlichen Embryo zeigt der Nerv im Allgemeinen dieselbe Structur wie bei dem $3,8 \mathrm{~cm}$ langen; an der Oberfläche der Papille macht sich eine leichte Finsenkung und die Arteria hyaloidea s. capsularis bemerkbar. Im Nerven selbst bemerkt man eine Arteria centralis. Die Bündel seiner Nervenfasern sind sehr deutlich.

Bei Säugethier-Embryonen von hoherem Alter nimmt der Nerv an Volumen zu und treten die Nervenbündel deutlicher hervor (beim Kaninchenembryo von $8,5 \mathrm{~cm}$ und beim ausgetragenen Kaninchen), während wir beim menschlichen Embryo von $21 \mathrm{~cm}$ die Papille entwickelt finden, die centralen Bündel sich sanft zurückbiegen und den Boden der leichten Einsenkung, welchen dieselbe zeigt, bilden helfen; der übrige Theil des Nerven verhält sich wie beim Erwachsenen. Die Scheiden des Sehnerven sind beinahe vollständig entwickelt. 
Beim neugebornen Kaninchen ist die Papille gebildet, ihre Einsenkung ist seicht, ihre Nervenbündel geben ihr die Gestalt, welche sie beim ausgewachsenen Kaninchen darbietet; doch sieht man von der Arteria centralis retinae ein Netz von Capillaren ausgehen, welche sich in das Ausbreitungsgebiet des Nerven (Fig. 9 e) hineinverlängern und ausserdem die Arteria hyaloidea s. capsularis (Fig. 9 f). Die Lamina cribrosa ist gebildet (Fig. $9 \mathrm{~g}$ ), die Nervenbündel im ubrigen Theil des Nerven sind auch sehr ausgeprägt und die Zellen, welche sie abgrenzen, zeigen einen Kern mit stark gefärbtem Reticulum; in diesen Elementen beobachtet man Karyokinese (Fig. $9 \mathrm{~h}$ ). Im Auge des neugebornen Menschen hat der Sehnerv seine Entwicklung vollendet.

Beim Kaninchen von 7 Tagen hat der Sehnerv und die Papille beinahe vollständig die Entwicklung wie im ausgewachsenen Zustand, doch sind die centralen Gefässe von einem jangen Bindegewebe mit Capillaren umgeben. Dasselbe beobachtet man bei einem Kaninchen von 15 Tagen; aber bei einem Kaninchen von 30-40-42 Tagen ist die Entwicklung des Sehnerven und seiner Scheiden vollendet.

Aus dem Stadium der Histogenese des Nervus opticus bei Säugethieren geht bervor, dass das Volumenwachsthum des Nervus opticus geschieht durch Vermehrung der zwischen den Nervenfaserbündeln liegenden Zellen vermittelst der Karyokinese und durch die Volumenzunahme der Bündel dieser Fibrillen.

Während bei einigen neugebornen Säugethieren (Mensch) die Papille und der übrige Theil des Sehnerven entwickelt sind wie beim Erwachsenen, zeigt bei anderen (Kaninchen) die Papille meist noch die Persistenz der Arteria hyaloidea s. capsularis.

Das Stützgewebe des Nervus opticus wird zum grossen Theil von den Zellen gebildet, welche die Wände seines 
Stieles darstellen, welche aber Veränderungen eingegangen sind, - aber auch von Elementen des Mesoderma, welche mit Gefässen in den Nerven eindringen.

Was ich beobachtete, berechtigt mich der Meinung von His und Kolliker in Betreff des Ursprungs des Nervus opticus beizutreten; darum halte ich daran fest, dass der Nervus opticus der Säugethiere als ein Theil des Gehirns zu betrachten ist und dass die Wandungen des Stiels der secundären Augenblase sich in Stützgewebe umwandeln, zu dessen Bildung auch das Mesoderma beiträgt, welches mit Gefässen hineinwächst. In der That kann man in diesem Stiel keinen Vorgang, der auf die Bildung von Nervenzellen hindeutete, beobachten, wie ich einen solchen bei der Bildung der Ganglienzellen verfolgen konnte; aus diesem Grunde können von den eigentlichen, den Stielwandungen des Nerven angehörigen Zellen die diesen Nerven wesentlich zusammensetzenden Nervenfibrillen nicht gebildet werden.

Gegen die Ansicht W. Müllers kann ich bemerken, dass das Auftreten der Nervenbündel des Sehnerven der Entwicklung der Ganglienzellen der Retina lange vorbergeht, diese Zellen daher den Sehnerven nicht bilden können, da er bereits vor ihnen existirt.

\section{Erklärung der Figuren.}

Fig. 1. Horizontaler Längsschnitt der secundären Augenblase eines 10 Tage alten Kaninchenembryo. - Färbung mit Grenachers Alauncarmin. - Koristka, Oc. 3. hochgestellter Tubus, Object VIII: a) Zellen in Karyokinese vom innern Rand des Sehnervenstiels; b) desgleichen von der inneren Oberfläche der proximalen Lamelle; c) desgleichen von der äusseren Oberflächenschicht der distasen Lamelle; d) desgleichen von dem die secundäre Augenblase umgebenden Mesoderma.

Fig. 2 A. Horizontaler Längsschnitt des hinteren Pols der secundüren Augenblase und der Anlage des Sehnerven von einem $1,8 \mathrm{~cm}$ langen Rindsembryo. - Färbung mit Grenachers Alaun- 
carmin. - Zeiss, Oc. 4, tiefgestellter Tubus, Obj. 1/12, homogene Immersion. a) Zellen der distalen Lamelle mit ovalem Kern; b) Zellen in Karyokinese in der äusseren Oberflächenschicht; c) desgleichen in der dritten Schicht der distalen Lamelle; d) Zellen der distalen in der Seitenansicht; e) Stelle, an welcher sich die Zellen der proximalen Lamelle in den Sehnervenstiel fortsetzen; f) Kerne der wahrscheinlich dem Mesoderma angehörigen Zellen, welche die Anlage des Nervus opticus mit der distalen Lamelle verbinden; g) feinste Nervenfibrille; h) Zellen in Karyokinese in dem Mesoderma, welches den Nerven und die secundäre Augenblase umgiebt.

Fig. 2 B. i) Zellen der proximalen Lamelle, von vorn gesehen. Hämatoxylinfärbung.

Fig. 3. Horizontaler Längsschnitt der distalen Lamelle am hintern Pole und des Nervus opticus von einem $1,8 \mathrm{~cm}$ langen Kaninchenembryo. - Fig. 3 A. Färbung mit Hämatoxylin. Fig. 3B. Färbung mit Grenachers Alanncarmin. Zeiss Oc.4, tiefgestellter Tubus, Obj. 1/12, homogene Immersion.

Fig. $3 \mathrm{~A}$. a) Zellen in Karyokinese in der zweitinnersten Schicht der distalen Lamelle; b) Karyokinese in den Zellen der Papille; c) Kernzellen mit gegen den Verlauf der Nervenfibrillen transversaler oder senkrechter Richtung; d) Karyokinese einiger Zellen; e) desgleichen in dem hintern Abschnitt des Sehnerven; h) mittlere Nervenfasern des Sehnerven; i) äussere Nervenfasern, welche im Begriff sind, sich gegen die innere Oberfläche der distalen Lamelle zurückzabiegen.

Fig. 3B. f) Zellen der proximalen Lamelle, von der Seite gesehen; g) dieselben Zellen in der Ansicht von vorn.

Fig. 4. Horizontalschnitt vom hintern Pol der distalen Lamelle der secundären Augenblase eines $4,2 \mathrm{~cm}$ langen Rindsembryo. - Färbung mit Grenachers Alauncarmin. - Zeiss, Oc. 4 , tiefgestellter Tubus, Obj. E. a) Zellen in Karyokinese in der äusseren Oberflächenschicht derselben; b) desgleichen in den mittleren Sehichten der äusseren Zone der distalen Lamelle; e) desgleichen in den inneren Schichten.

Fig. 5. Horizontalschnitt der distalen Lamelle desselben Enbryo wie in Fig. 4 an der Grenze zwischen der Aequatorialgegend und der Pars ciliaris retinae. Färbung mit Grenachers Alauncarmin. - Zeiss, Oc.4, tiefgestellter Tabus, Obj. E. a) Zellen in Karyokinese in der änsseren Oberlächensehicht; b) desgleichen in der mittleren Partie der distalen Lamelle.

Fig. 6. Horizontaler Längsschnitt der distalen Lamelle der secundären Augenblase von einem $2,7 \mathrm{~cm}$ langen Kaninchenembryo. Zeiss, Oc. 4, tiefgestellter Tubus, Obj. 1/12, homogene Immersion. Fig. 6 A Färbung mit Hämatoxylin, Fig. 6 B Färbung mit Alauncarmin. 
Fig. 6 A. a) Centrale Nervenbündel des Sehnerven; b) periphere Nervenbündel des Opticus, welche sich strahlenförmig zurückbiegen; e) Zellen in Karyokinese im Anfang der Sehnervenansbreitung; d) senkrecht zur Riohtung der Nervenbündel gestellte Zellkerne und einige davon in Karyokinese; e) längliche und spindelförmige Zellkerne in der hintern Partie des Nervus opticus und einige dieser Zellen in Karyokinese; f) Karyokinese in einer Zelle der zweiten Schicht der dunkeln Aussenzone der distalen Lamelle; g) Zellen der hellen Innenzone; h) Tapetum in Seitenansicht; i) Karyokinese der Zellen des Mesoderma, welches den Nerv umgiebt.

Fig. 6B. 1) Tapetum von vorn gesehen; 1) cubische Zellen des Tapetum in Seitenansicht und einige derselben in Raryokinese.

Fig. 7. Theil eines Horizontalschnitts der distalen Lamelle von einem 4,3 cm langen Kaninchenembryo. - Zeiss, Oc. 4, tiefgestellter Tubus, Obj. 1/22, homogene Immersion. Fürbung mit Hämatoxylin. a) Bildungszellen der donkeln Aussenzone; b) Beginn der innern reticulären Schicht; c) Zellen der hellen Innenzone; d) desgleichen in Karyokinese; e) Zellen zwischen den Nervenfibrillen der Sehnervenausbreitung in Karyokinese.

Fig. 8. Theil eines Horizontalschnitts der distalen Lamelle am hintern Pole von einem 5,7 cm langen Kaninchenembryo, Färbung nach der Methode von Bizzozero. - Zeiss, Oc. 4, tiefgestellter Tubus, Obj. 1/12, homogene Immersion. a) Zellen der dunkeln Aussenzone mit ovalem Kern an der Grenze gegen die innere reticuläre Sehicht; b) Stratum reticulare internum; c) Zellen der hellen Innenzone, eine derselben d) in Karyokinese.

Fig. 9. Horizontaler Längsschnitt der Retina an ihrem hinteren Pole und des Nervus opticus vom neugeborenen Kaninchen. Färbung mit Grenachers Alaunearmin. - Zeiss, Oc. 4, tiefgestellter Tubus, Obj. $1 / 12$, homogene Immersion. a) Zellen in Karyokinese aus der äusseren Oberflächenschicht der distalen Lamelle; b) ovale farblose Zellen an der äusseren Grenze der Aussenzone; c) ovale und runde Zellen zwischen den Ganglienzellen; d) Ganglienzellen; e) Capillare in der Sehnervenausbreitung; f) Arteria hyaloidea s. capsularis; g) Lamina cribrosa; b) Karyokinesis in den Zellen des Sehnerven; i) Epithel der Retina in Seitenansicht.

Fig. 10. Horizontaler Lüngsschnitt der distalen Lamelle am hintern Pole, und des Nervus opticus von einem $3,8 \mathrm{~cm}$ langen menschlichen Embryo. Färbung mit Grenachers Alauncarmin. Zeiss, Oc. 4, tiefgestellter Tubus, Obj. $1 / 2$, homogene Immersion.

Fig. $10 \mathrm{~A}$. a) Dunkle Aussenzone; b) Karyokinesis in der äusseren Oberfächenschicht; c) Zellen mit grossen blassen Kernen der hellen Innenzone; d) centrale Nervenbündel der Papille; e) periphere Nervenbündel, welche sich strahlenförmig zurückbiegen; 
Fr. Falchi.

f) Zellen in Karyokinese im Nervus opticus; g) Epithel der proximalen Lamelle in Seitenansicht.

Fig. 10 B. h) Zellen des Tapetum in der Ansicht von vorn. Fig. 11. Horizontalsehnitt der Retina in der Aequatorialgegend eines 21,5 cm langen menschlichen Embryo. - Färbung mit Grenachers Alauncarmin. Zeiss, Oc. 4, tiefgestellter Tubus, Obj. $1 / 12$, homogene Immersion.

Fig. $11 \mathrm{~A}$. a) Membrana limitans externa; b) Verlängerung des Kerns hei der Entwicklung der Stäbchen; c) die Kerne, welehe sich zur Bildung der Stäbchen verlängern, sind durch die Membrana limitans externa durchgewachsen; d) vergrösserte und verlängerte Keme bei der Entwicklung der Zapfen nähern sich der Membrana limitans externa und erreichen dieselbe; e) diese Kerne haben die Membrana limitans externa durchsetzt und zeigen nun die Gestalt eines gestielten ovalen Bläschens; f) Zellen mit kleinem runden ovalen Kern, welcher sich intensiv färbt; g) Zellen mit grossem, blassen, ovalen Kexn; h) bipolare, polypolare und spindelförmige Z ellen; i) grosser blasser Kern im Stratum reticulare internnm; k) grosser Zellkern der weiter innen gelegenen Schicht; l) kleiner Zellkern der weiter innen gelegenen Schicht; m) Schicht der Nervenfibrillen; n) inneres Ende der Müller'schen Radialfasern; o) Epithel der Retina in Seitenansicht.

Fig. 11 B. p) Epithel der Retina in der Ansicht von vorn. 


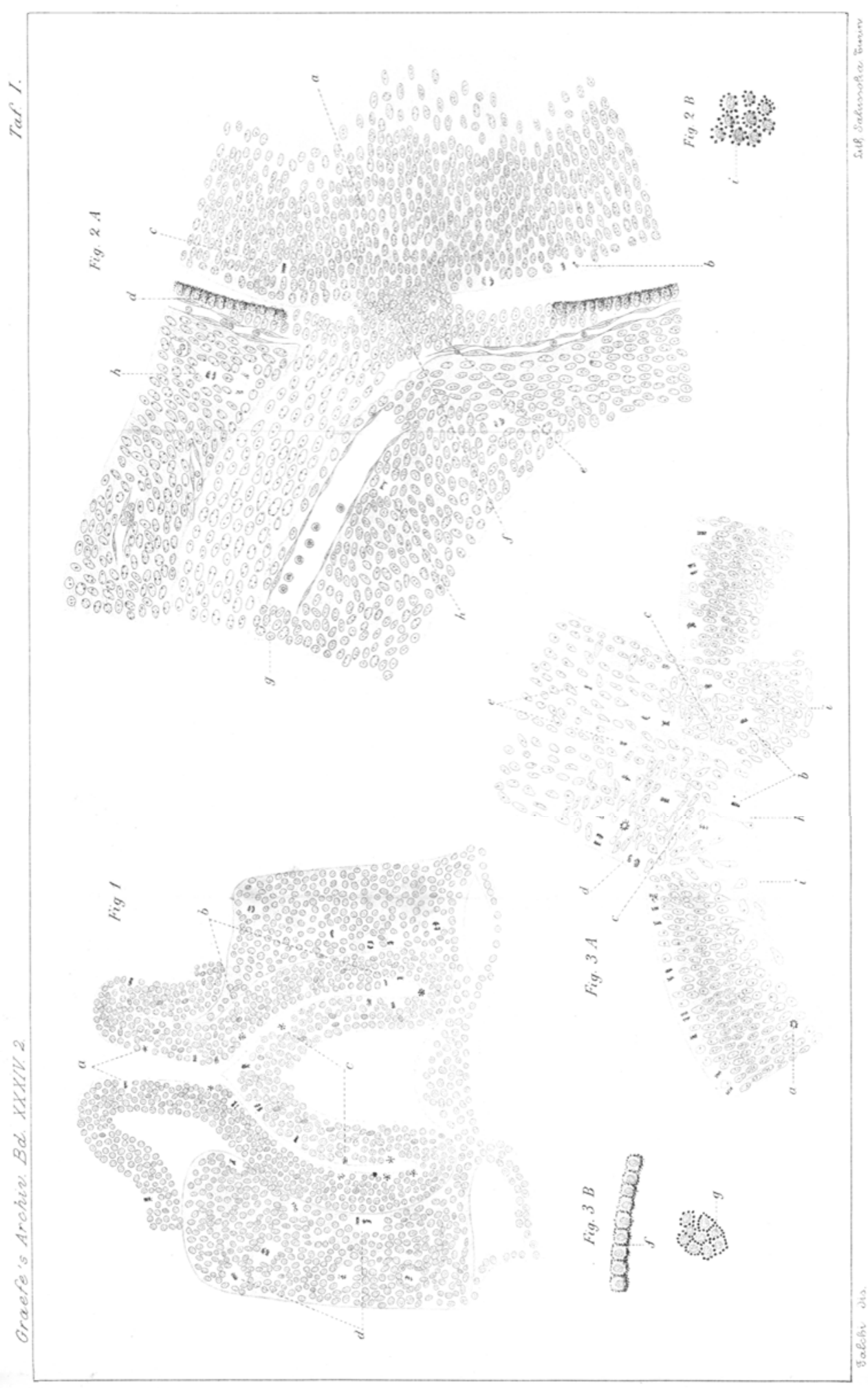



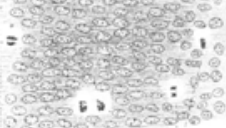

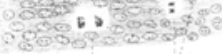

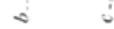

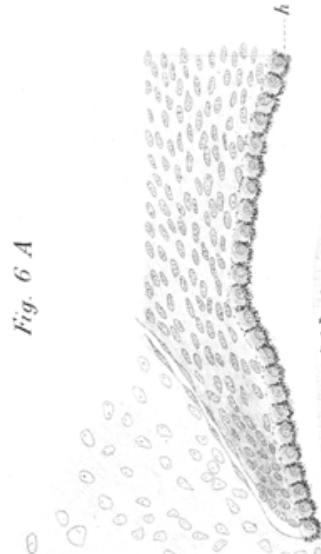
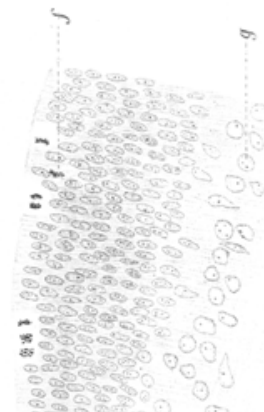

$\cos \theta \sin \theta 0$
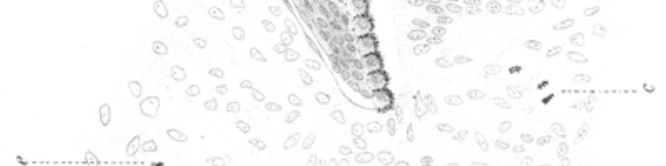

का

180
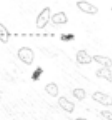

Sent on

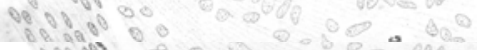

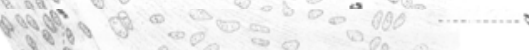

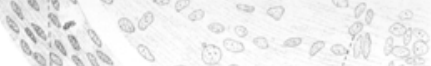

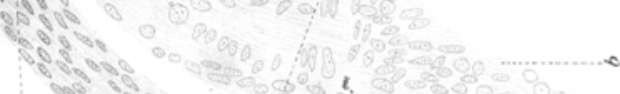

\&

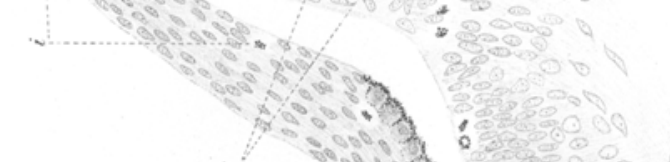



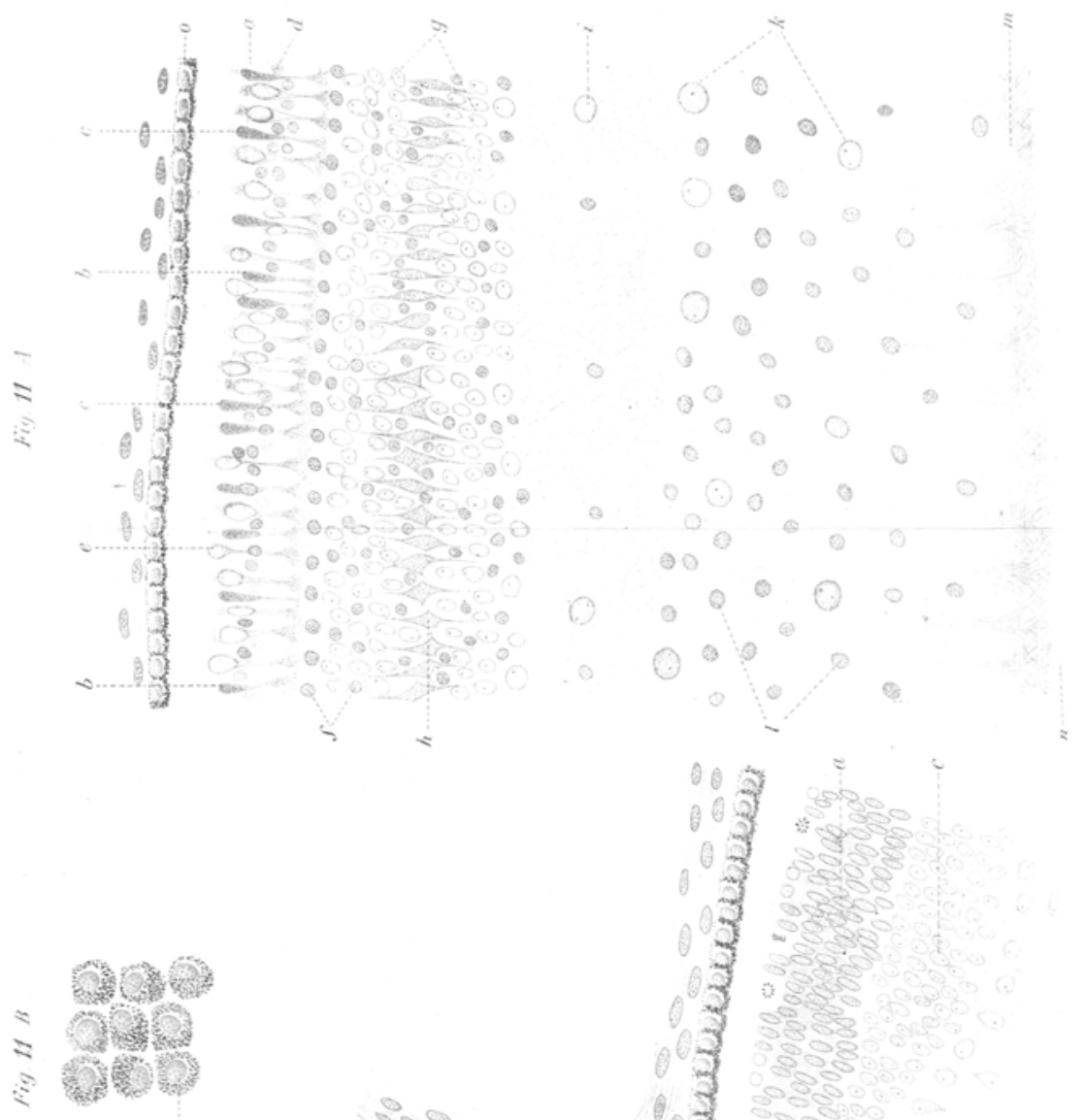

$$
\text { son }
$$

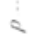

80

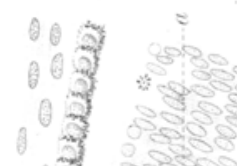




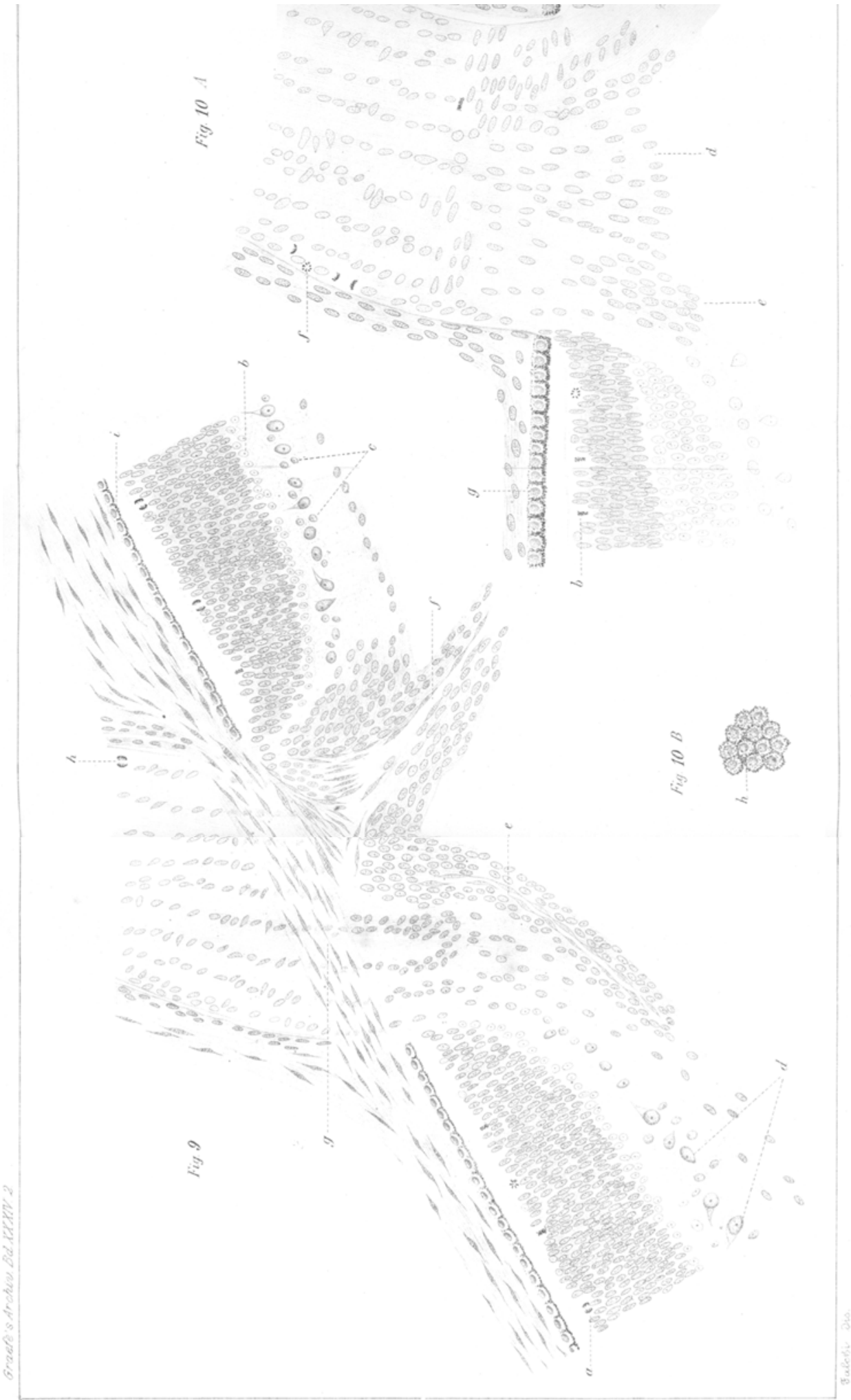

